\title{
Vanishing of Vacuum States and Blow-up Phenomena of the Compressible Navier-Stokes Equations
}

\author{
Hai-Liang $\mathbf{L I}^{1,3}$, Jing $\mathbf{L I}^{2,3}$, Zhouping $\mathbf{X I N}^{3,1}$ \\ ${ }^{1}$ Department of Mathematics, Capital Normal University \\ Beijing, P. R. China, email: hailiang_li@mail.cnu.edu.cn \\ ${ }^{2}$ Institute of Applied Mathematics, AMSS, Academia Sinica \\ Beijing 100080, P. R. China, email: ajingli@gmail.com \\ ${ }^{3}$ Institute of Mathematical Science, The Chinese University of Hong Kong \\ Shatin, Hong Kong, email: zpxin@ims.cuhk.edu.hk
}

\begin{abstract}
The Navier-Stokes systems for compressible fluids with density-dependent viscosities are considered in the present paper. These equations, in particular, include the ones which are rigorously derived recently as the Saint-Venant system for the motion of shallow water, from the Navier-Stokes system for incompressible flows with a moving free surface [14]. These compressible systems are degenerate when vacuum state appears. We study initial-boundary-value problems for such systems for both bounded spatial domains or periodic domains. The dynamics of weak solutions and vacuum states are investigated rigorously.

First, it is proved that the entropy weak solutions for general large initial data satisfying finite initial entropy exist globally in time. Next, for more regular initial data, there is a global entropy weak solution which is unique and regular with well-defined velocity field for short time, and the interface of initial vacuum propagates along particle path during this time period. Then, it is shown that for any global entropy weak solution, any (possibly existing) vacuum state must vanish within finite time. The velocity (even if regular enough and well-defined) blows up in finite time as the vacuum states vanish. Furthermore, after the vanishing of vacuum states, the global entropy weak solution becomes a strong solution and tends to the non-vacuum equilibrium state exponentially in time.
\end{abstract}




\section{Introduction}

The compressible isentropic Navier-Stokes equations, which are the basic models describing the evolution of a viscous compressible fluid, read as follows

$$
\left\{\begin{array}{l}
\rho_{t}+\operatorname{div}(\rho u)=0, \\
(\rho u)_{t}+\operatorname{div}(\rho u \otimes u)-2 \operatorname{div}(\mu D(u))-\nabla(\xi \operatorname{div} u)+\nabla p(\rho)=0
\end{array}\right.
$$

where $x \in \Omega \subset R^{N}, t \in(0, T), D(u)=\left(\nabla u+(\nabla u)^{\mathrm{T}}\right) / 2$, and $p(\rho)=a \rho^{\gamma}, a>0, \gamma \geq 1$, the viscosity coefficients $\mu, \xi$ are assumed to satisfy $\mu \geq 0$ and $\xi+2 \mu / N \geq 0$.

If $\mu$ and $\xi$ are both constants, there is huge literature on the studies of the global existence and behavior of solutions to (1.1). For instance, the one-dimensional (1D) problems were addressed by Kazhikhov et al [24] for sufficiently smooth data, and by Serre [46,47] and Hoff [18] for discontinuous initial data, where the data are uniformly away from the vacuum; the multidimensional problems (1.1) were investigated by Matsumura et al [35-37], who proved global existence of smooth solutions for data close to a non-vacuum equilibrium, and later by Hoff for discontinuous initial data [19], and more recently, by Danchin [11], who obtained existence and uniqueness of global solutions in a functional space invariant by the natural scaling of the associated equations; and for the existence of solutions for arbitrary data(which may include vacuum states), Lions [29-31] (see also Feireisl et al [13]) obtained global existence of weak solutions defined as solutions with finite energy - when the exponent $\gamma$ is suitably large, where the only restriction on initial data is that the initial energy is finite, so that the density is allowed to vanish.

Despite the important progress, the regularity, uniqueness and behavior of these weak solutions remain largely open. As emphasized in many papers related to compressible fluid dynamics $[9,10,18,20,21,24,27,45,46,48,50,52]$, the possible appearance of vacuum is one of the major difficulties when trying to prove global existence and strong regularity results. Hoff and Smoller [21] proved that weak solutions of the compressible Navier-Stokes equations (1.1) in one space dimension do not exhibit vacuum states in a finite time provided that no vacuum is present initially under fairly general conditions on the data. Such a result was extended to the spherically symmetric case in [54] recently. On the other hand, the results of Xin [52] showed that there is no global smooth solution to Cauchy problem for (1.1) with a nontrivial compactly supported initial density, which gives results for finite time blow-up in the presence of vacuum. It is also proved in [27] that for bounded domain even one point initial vacuum shall cause the global strong solutions to 1D (1.1) to blow up as time goes to infinity provided the initial data satisfies some compatibility conditions.

The independence of viscosities on density makes it possible to trace either the particle path or the trajectory of vacuum state. This, however, leads to the failure of continuous dependence of weak solutions containing vacuum state on initial data [20]. For the case that the density changes continuously across the interfaces separating the gas and vacuum, the global existence and uniqueness of weak solutions was obtained 
in [33], where the authors obtained that velocity is smooth enough up to the interfaces which are particle paths separating the gas from the vacuum and that the support of gas density expands outside as well as interface connecting gas and vacuum moves at an algebraic rate.

Thus, viscous compressible fluids near vacuum should be better modeled by the compressible Navier-Stokes equations with density-dependent viscosities, as was derived in the fluid-dynamical approximation of Boltzmann equation for dilute gases. Further, as was first pointed out and investigated by Liu-Xin-Yang in [32], in the derivation of the compressible Navier-Stokes equations from the Boltzmann equation by the Chapman-Enskog expansions, the viscosity depends on the temperature, which is translated into the dependence of the viscosity on the density for isentropic flows. Moreover, it should be emphasized that a one-dimensional compressible flow model, called the viscous Saint-Venant system for laminar shallow water, derived rigorously from incompressible Navier-Stokes system with a moving free surface by Gerbeau-Perthame recently in [14], has the form:

$$
\left\{\begin{array}{l}
\rho_{t}+(\rho u)_{x}=0 \\
(\rho u)_{t}+\left(\rho u^{2}\right)_{x}-a\left(\rho u_{x}\right)_{x}+\left(\rho^{2}\right)_{x}=0
\end{array}\right.
$$

with the viscosity coefficients given by $\mu(\rho)=\xi(\rho)=a \rho / 3$ for a given positive constant $a$. Indeed, such models appear naturally and often in geophysical flows $[2,6,7]$.

In the case of one-dimensional problem with $\mu=\xi=a \rho^{\alpha}$ for some positive constants $a$ and $\alpha$, the well-posedness of the Cauchy problem has been studied by many authors for not only initially compact-supported density but initially non-vacuum density. Indeed, for the initially compact-supported density case, the local (in time) well-posedness of weak solutions to this problem was first established by Liu-Xin-Yang in [32], where the initial density was assumed to be connected to vacuum with discontinuities. This property, as shown in [32], can be maintained for some finite time. And the global existence of weak solutions, together with $\alpha \in(0,1)$ and the density function connecting to vacuum with discontinuities, was considered by many authors, see $[23,43,55]$ and the references therein. It is noticed that the above analysis is based on the uniform positive lower bound of the density with respect to the construction of the approximate solutions. On the other hand, if the density function connects to vacuum continuously, there is no positive lower bound for the density and the viscosity coefficient vanishes at vacuum. This degeneracy in the viscosity coefficient gives rise to new difficulties in analysis because of the less regularizing effect on the solutions. Yang et al [56] first obtained a local existence result for this case under the free boundary condition with $\alpha>1 / 2$. The authors in $[12,51,57]$ obtained the global existence of weak solutions with $\alpha \in(0,1 / 2)$.

However, almost all above results concern mainly with free boundary problems, and for the global existence results, the choices of viscosity do not fit the important physical model, the shallow water equation (1.2) with $\mu(\rho)=\rho$ (namely $\alpha=1$ ). For the constant viscosity case, one has known not only that the vacuum state will not develop 
later on time if there is no vacuum state initially [21], but also that the separate two initial vacuum states shall not meet together in a finite time [54], and that one point initial vacuum causes strong solutions to blow up [27] at infinity as well. However, little is known on the dynamics of the vacuum states of weak solutions to the compressible Navier-Stokes equations (1.1) with density-dependent viscosity on bounded domain. And in particular, it is not clear yet how the vacuum states evolve with respect to time and whether the initial vacuum states shall exist all the time or not for weak solutions. The study of these important dynamical problems about vacuum states is rather difficult because the nonlinear diffusion is degenerate as vacuum appears, which is quite different from the case of constant viscosity. This causes the loss of information about the velocity and makes it difficult to trace the evolution of vacuum states in general. Across the interface (or vacuum boundary), it is usually difficult to obtain enough information about velocity even if considering specific cases such as point vacuum or continuous vacuum of one piece. It is important to get enough information about the velocity since the flow particles transport usually along particle path, and all interfaces of vacuum, such as free boundaries [23,33] which can be observed and dealt with, also move along the trajectories determined by velocity field.

For the multidimensional case, Vaigant et al [49] first proved that for the 2D case and for the case $\mu$ is a constant and $\xi(\rho)=a \rho^{\beta}$, with $a>0, \beta>3$, (1.1) with periodic boundary condition has a unique strong and classical solution with density away from vacuum. More recently, Bresch and Desjardins [2,3,6,7] (see also [38]) have made important progress. Under the condition that $\xi(\rho)=2\left(\mu^{\prime}(\rho) \rho-\mu(\rho)\right)$, they establish a new Bresch-Desjardins (BD) entropy inequality which can not only be applied to the vacuum case but also used to get the compactness results for (1.1) which extended the compactness results due to Lions [29-31] to the case $\gamma \geq 1$. On the other hand, the constructions of the approximation solutions does not seem routine in the general case of appearance of vacuum. However, it should be noted that recently Bresch et al [4-6] have made significant progress on the construction of approximate solutions and existence of global weak solutions to the multi-dimensional compressible NavierStokes equations and the 2D shallow water model in the case that there is either a drag friction (for barotropic compressible flows) or a cold pressure (for viscous and heat conducting flows). In the case that there is neither drag friction nor cold pressure included, Guo-Jiu-Xin [16] recently have shown how to construct approximate smooth solutions and obtain the global existence of weak solutions via the BD entropy to the (2D and 3D) barotropic compressible Navier-Stokes for the spherical symmetric initial data. There are also other recent interesting applications of the BD entropy to one-dimensional compressible Navier-Stokes equations with degenerate viscosities, for instance, on the global existence and long time behavior of weak solutions for free boundary problem $[15,17]$, or the existence and uniqueness of global strong solution away from vacuum in real line [39].

We study mainly the initial-boundary-value problem (IBVP) for (1.1), where $\mu=\rho^{\alpha}$ with $\alpha>1 / 2$, on spatial one-dimensional bounded spatial domains or periodic domains. This contains the physical important model for shallow water equations (1.2). The 
choice of $\alpha>1 / 2$ is necessary in order to consider the dynamics of vacuum states since it allows the existence of initial vacuum in Eulerian coordinates as one can see later.

We first establish the global existence of entropy weak solutions for the compressible Navier-Stokes equations (1.1), with pressure $p=\rho^{\gamma}$ and $\gamma \geq \alpha / 2$, for general initial data with finite entropy and vacuum. The key in our analysis is the construction nonvacuum approximate solutions so that we can make use of the stability analysis in [38], where the Bresch-Desjardins (BD) entropy inequality (see $[2,3,6,7]$ ) was used to obtain the compactness results. Our construction of the approximate solutions is strongly motivated by the previous work of Jiang-Xin-Zhang [23] about the existence of global weak solutions to one-dimensional compressible Navier-Stokes with free boundary as vacuum interface. In general, it seems rather difficult to investigate the dynamics of vacuum states due to the degeneracy of nonlinear diffusion and the density function connecting to vacuum continuously. Therefore, we further consider the cases of more regular initial data containing point vacuum or continuous vacuum of one piece, and we show that there is a global entropy weak solution which is unique and regular with well-defined velocity field at least for short time, and the vacuum states remain for the short time. Then, we use some ideas due to $[22,27,28]$ to prove that any possible vacuum state in such global weak solutions which satisfy the BD entropy must vanish within finite time. This shows that such short time structure and vacuum states of weak solutions can not be maintained all the time. And as the vacuum states vanish, the spatial derivative of velocity (if it exists) has to blow up even if the velocity is regular enough and well-defined before. After the vanishing of vacuum states, we can redefine the velocity field and recover the nonlinear diffusion term in terms of density and velocity. In addition, the global entropy weak solution is shown to become a strong solution and tends to the non-vacuum equilibrium state exponentially in time. This phenomena, applied to the compressible shallow water equations (1.2), seems to be never observed for the compressible Navier-Stokes equations before.

The rest of the paper is as follows. In section 2, the main results about the vanishing of vacuum states and blow-up phenomena of global entropy weak solutions for the compressible Navier-Stokes equations are stated. The global existence of entropy weak solutions for general large initial data with vacuum states allowed is proven in section 3 . The short time structure of global entropy weak solution with initial one point vacuum state or initial continuous vacuum states of one piece are investigated in section 4 . In section 5, we show the vanishing of vacuum states and blow-up phenomena of any global entropy weak solution within finite time and analyze the regularity and large time asymptotic behavior of global entropy weak solutions after the vanishing of vacuum states. 


\section{Main results}

We consider the initial-boundary-value-problem (IBVP) for the 1D compressible Navier-Stokes equations with density-dependent viscosity

$$
\begin{gathered}
\rho_{t}+(\rho u)_{x}=0 \\
(\rho u)_{t}+\left(\rho u^{2}+p(\rho)\right)_{x}-\left(\mu(\rho) u_{x}\right)_{x}=0
\end{gathered}
$$

with $\rho \geq 0$ the density, $\rho u$ the momentum. The pressure and viscosity are assumed to have the form:

$$
p(\rho)=a_{1} \rho^{\gamma}, \mu(\rho)=a_{2} \rho^{\alpha}
$$

where $\gamma \geq 1, a_{1}>0, a_{2}>0$, and $\alpha>1 / 2$ are constants, and for simplicity we set $a_{1}=a_{2}=1$.

The initial data is given for the density $\rho$ and the momentum $\rho u$

$$
\rho(x, 0)=\rho_{0}(x) \geq 0, \quad \rho u(x, 0)=m_{0}(x), \quad x \in \Omega,
$$

where the domain $\Omega$ is chosen as unit interval denoting the spatial domain $(0,1)$ or periodic domain with period length one, and throughout the present paper the initial data is assumed to satisfy

$$
\left\{\begin{array}{l}
\rho_{0} \geq 0 \text { a.e. in } \Omega, \quad \rho_{0} \in L^{1}(\Omega), \quad\left(\rho_{0}^{\alpha-1 / 2}\right)_{x} \in L^{2}(\Omega), \\
m_{0}=0, \text { a.e. on }\left\{x \in \Omega \mid \rho_{0}(x)=0\right\}, \frac{\left|m_{0}\right|^{2}}{\rho_{0}} \in L^{1}(\Omega) .
\end{array}\right.
$$

Remark 2.1 Note here that the condition (2.4) implies

$$
\rho_{0} \in L^{\infty}(\Omega), \quad \rho_{0} \log _{+} \rho_{0} \in L^{1}(\Omega)
$$

It should be clear that a large class of initial data satisfy the conditions in (2.4). In particular, the assumptions (2.4) are satisfied for following initial data

$$
\rho_{0}(x)=\left(\left|x-x_{0}\right|^{2}\right)^{1 /(2 \alpha-1)}, \quad m_{0}(x)=0, \quad x \in \Omega .
$$

Without the loss of generality, the total initial mass is renormalized to be one throughout the present paper, i.e.,

$$
\int_{\Omega} \rho_{0}(x) d x=1
$$

The boundary conditions are one of the boundary conditions of Dirichlet type and periodic type for Eqs. (2.1)-(2.2) imposed as

(1). Dirichlet case:

$$
\rho u(0, t)=\rho u(1, t)=0, \quad t \geq 0,
$$


(2). periodic case:

$$
\rho, u \text { are periodic in } x \text { of period } 1 \text {, }
$$

where we consider Eqs. (2.1)-(2.2) on $\mathbb{R} \times(0, \infty)$.

Remark 2.2 For the case of Dirichlet boundary (2.6), the boundary is given by the physical observable momentum instead of velocity. It is natural to employ such boundary condition as considering the dynamics of (global in time) weak solutions to the IBVP for the compressible Navier-Stokes equations with possible vacuum states included since it is usually the case that, at vacuum states, the momentum is zero and is observed and controllable, but almost nothing is known yet for the velocity for weak solutions. Note here that for any (weak) solution away from vacuum at the boundary, the boundary condition (2.6) reduces to $u(0, t)=u(1, t)=0, t \geq 0$.

In order to define the weak solutions to the IBVP for the compressible Navier-Stokes equations (2.1) - (2.2) with initial data (2.3) and boundary condition (2.6) or (2.7), we define the set of test functions as follows,

$$
\Psi \triangleq \begin{cases}C_{0}^{\infty}(\bar{\Omega} \times[0, T)) & \text { for the Dirichlet case (2.6) } \\ C_{\text {per }}^{\infty}(\mathbb{R} \times[0, T)) & \text { for the periodic case (2.7) }\end{cases}
$$

and

$$
\Phi \triangleq \begin{cases}C_{0}^{\infty}(\Omega \times[0, T)) & \text { for the Dirichlet case (2.6) } \\ C_{\text {per }}^{\infty}(\mathbb{R} \times[0, T)) & \text { for the periodic case (2.7) }\end{cases}
$$

with $C_{\text {per }}^{\infty}(\mathbb{R} \times[0, T))$ defined by

$$
C_{\text {per }}^{\infty}(\mathbb{R} \times[0, T))=\left\{\varphi \in C^{\infty}(\mathbb{R} \times[0, T)) \mid \varphi \text { is periodic in } x \text { of period } 1\right\} .
$$

We define the weak solutions to the IBVP for the compressible Navier-Stokes Equations (2.1)-(2.2) as follows.

Definition 2.3 (global weak solutions) For any $T>0,(\rho, u)$ is said to be a weak solution to Eqs. (2.1) -(2.2) with initial data (2.3) and boundary value (2.6) or (2.7) in $\Omega \times(0, T)$, if

$$
\left\{\begin{array}{l}
0 \leq \rho \in L^{\infty}\left(0, T ; L^{1}(\Omega) \cap L^{\gamma}(\Omega)\right), \quad\left(\rho^{\alpha-1 / 2}\right)_{x} \in L^{\infty}\left(0, T ; L^{2}(\Omega)\right) \\
\sqrt{\rho} u \in L^{\infty}\left(0, T ; L^{2}(\Omega)\right), \quad \rho^{\alpha} u_{x} \in L^{2}\left(0, T ; W_{\mathrm{loc}}^{-1,1}(\Omega)\right)
\end{array}\right.
$$

and $(\rho, u)$ satisfies

$$
\int_{\Omega} \rho_{0} \psi(x, 0) d x+\int_{0}^{T} \int_{\Omega} \rho \psi_{t} d x d t+\int_{0}^{T} \int_{\Omega} \sqrt{\rho} \sqrt{\rho} u \psi_{x} d x d t=0
$$


for any $\psi \in \Psi$, and

$$
\begin{aligned}
\int_{\Omega} m_{0} \varphi(x, 0) d x+\int_{0}^{T} \int_{\Omega} \sqrt{\rho}(\sqrt{\rho} u) \varphi_{t} d x d t \\
\quad+\int_{0}^{T} \int_{\Omega}\left((\sqrt{\rho} u)^{2}+\rho^{\gamma}\right) \varphi_{x} d x d t-\left\langle\rho^{\alpha} u_{x}, \varphi_{x}\right\rangle=0
\end{aligned}
$$

for all $\varphi \in \Phi$. The nonlinear diffusion term $\rho^{\alpha} u_{x}$ is defined as

$$
\begin{aligned}
\left\langle\rho^{\alpha} u_{x}, \varphi\right\rangle= & -\int_{0}^{T} \int \rho^{\alpha-1 / 2} \sqrt{\rho} u \varphi_{x} d x d t \\
& -\frac{2 \alpha}{2 \alpha-1} \int_{0}^{T} \int\left(\rho^{\alpha-1 / 2}\right)_{x} \sqrt{\rho} u \varphi d x d t
\end{aligned}
$$

for any $\varphi \in \Phi$, where $\rho \in L^{\infty}(\Omega \times(0, T))$ due to (2.8). Moreover, for the spatial periodic case (2.7), $(\rho, \sqrt{\rho} u)$ is also periodic.

Remark 2.4 For the Dirichlet case, (2.9), together with the fact $\rho \in L^{\infty}(\Omega \times(0, T))$ due to (2.8), implies that $(\rho, u)$ satisfies the Eq. (2.1) in the sense of distribution and justifies the boundary condition (2.6) in the sense that, for any time interval $I \subset[0, T]$,

$$
\varepsilon^{-1} \int_{I} \int_{0}^{\varepsilon} \sqrt{\rho} \sqrt{\rho} u(x, s) d x d s \rightarrow 0, \quad \varepsilon^{-1} \int_{I} \int_{1-\varepsilon}^{\varepsilon} \sqrt{\rho} \sqrt{\rho} u(x, s) d x d s \rightarrow 0
$$

as $\varepsilon \rightarrow 0^{+}$. If further $\sqrt{\rho} \sqrt{\rho} u \in L^{p}\left(0, T ; W^{1, q}(\Omega)\right)$ for some $p \geq 1, q \geq 1$, then (2.9) yields that $\sqrt{\rho} \sqrt{\rho} u \in L^{p}\left(0, T ; W_{0}^{1, q}(\Omega)\right)$, that is, the Dirichlet boundary condition (2.6) is satisfied in the sense of trace.

Definition 2.5 (global entropy weak solutions) Let $(\rho, u)$ be a global weak solution (in the sense of Definition [2.3) to (2.1) -(2.2) with initial data (2.3) and boundary value (2.6) or (2.7) in $\Omega \times(0, T)$. Then, $(\rho, u)$ is said to be a global entropy weak solution if there exists some function $\Lambda \in L^{2}(\Omega \times(0, T))$ satisfying (2.11), i.e.,

$$
\begin{aligned}
\int_{0}^{T} \int \Lambda \varphi d x d t= & -\int_{0}^{T} \int \rho^{\alpha-1 / 2} \sqrt{\rho} u \varphi_{x} d x d t \\
& -\frac{2 \alpha}{2 \alpha-1} \int_{0}^{T} \int\left(\rho^{\alpha-1 / 2}\right)_{x} \sqrt{\rho} u \varphi d x d t
\end{aligned}
$$

for any $\varphi \in \Phi$, and the following uniform entropy inequality holds

$$
\begin{aligned}
& \sup _{0 \leq t \leq T} \int_{\Omega}\left(|\sqrt{\rho} u|^{2}+\left|\left(\rho^{\alpha-1 / 2}\right)_{x}\right|^{2}+\pi(\rho)\right)(x, t) d x \\
& +\int_{0}^{T} \int_{\Omega}\left(\left|\left(\rho^{(\gamma+\alpha-1) / 2}\right)_{x}\right|^{2}+\Lambda^{2}\right)(x, t) d x d t
\end{aligned}
$$




$$
\leq C_{0} \int_{\Omega}\left(\frac{\left|m_{0}\right|^{2}}{\rho_{0}}+\left|\left(\rho_{0}^{\alpha-1 / 2}\right)_{x}\right|^{2}+\pi_{+}\left(\rho_{0}\right)\right)(x) d x
$$

with $C_{0}>0$ independent of $T$, and

$$
\pi(\rho) \triangleq\left\{\begin{array} { l l } 
{ \rho \operatorname { l o g } \rho , } & { \text { if } \quad \gamma = 1 , } \\
{ \frac { 1 } { \gamma - 1 } \rho ^ { \gamma } , } & { \text { if } \quad \gamma > 1 , }
\end{array} \quad \pi _ { + } ( \rho ) \triangleq \left\{\begin{array}{lll}
\rho \log _{+} \rho, & \text { if } \quad \gamma=1, \\
\frac{1}{\gamma-1} \rho^{\gamma}, & \text { if } \quad \gamma>1 .
\end{array}\right.\right.
$$

We have the following result on the existence of global entropy weak solutions.

Theorem 2.1 (Global existence) Assume that

$$
\alpha>\frac{1}{2}, \quad \gamma>\frac{\alpha}{2} .
$$

and that the initial data $\left(\rho_{0}, m_{0}\right)$ satisfies (2.4) and $\frac{\left|m_{0}\right|^{2+\nu}}{\rho_{0}^{1+\nu}} \in L^{1}(\Omega)$ for some positive constant $\nu$. Then for any $T>0$, there exists a global entropy weak solution $(\rho, u)$ to the IBVP for the compressible Navier-Stokes equations (2.1)-(2.3) with boundary condition (2.6) or 2.7) in $\Omega \times(0, T)$ in the sense of Definition 2.5.

Moreover, for the case of Dirichlet boundary condition (2.6), if $\alpha \in(1 / 2,3 / 2)$ and $\nu$ satisfies [2.17) (see Remark 2.6 below), then in addition to (2.8), the solution $(\rho, \sqrt{\rho} u)$ satisfies

$$
\sqrt{\rho}(\sqrt{\rho} u) \in L^{2}\left(0, T ; W_{0}^{1,(4+2 \nu) /(4+\nu)}(\Omega)\right),
$$

i.e., $\sqrt{\rho}(\sqrt{\rho} u)$ satisfies the Dirichlet boundary (2.6) in the sense of trace.

Remark 2.6 (1). Theorem 2.1 above holds for the compressible shallow water equation (1.2).

(2). For the Dirichlet case (2.6), one of the available ways (available for the case $\alpha \in(1 / 2,3 / 2)$ and $\gamma \geq 1)$ to obtain that $\sqrt{\rho} \sqrt{\rho} u \in L^{p}\left(0, T ; W^{1, q}(\Omega)\right)$, for some $p \geq$ $1, q \geq 1$, is to choose the positive constant $\nu$ in Theorem 2.1 such that

$$
\left\{\begin{array}{lll}
\nu \in(0,2 \gamma-\alpha), & \text { for } \alpha \in\left(\frac{1}{2}, 1\right], & \gamma \geq 1, \\
\nu \in\left[\frac{2(\alpha+\gamma-2)}{3-\alpha-\gamma}, \frac{2(2 \gamma-\alpha)}{1+\alpha-2 \gamma}\right], & \text { for } \alpha \in\left(1, \frac{3}{2}\right), & \gamma \in\left[1, \frac{1+\alpha}{2}\right), \\
\nu \in\left[\frac{4(\alpha-1)}{3-2 \alpha}, \infty\right), & \text { for } \alpha \in\left(1, \frac{3}{2}\right), & \gamma \geq \frac{1+\alpha}{2} .
\end{array}\right.
$$

Next, we show that there is a global entropy weak solution $(\rho, u)$ in the sense of Definition 2.5 for which the vacuum states and the structure of interface, if existing initially, can be preserved for a short time, so long as the initial data has additional regularity besides (2.4) and the fluids and the vacuum states in initial data are connected "smoothly". In addition, the weak solution $(\rho, u)$ is actually a unique regular solution for the short time. For simplicity, we consider the case of one point vacuum state 
contained at $x=x_{0} \in(0,1)$ in the initial data $\left(\rho_{0}, m_{0}\right)=\left(\rho_{0}, \rho_{0} u_{0}\right)$ with additional regularity

$$
\begin{gathered}
A_{0}\left|x-x_{0}\right|^{\sigma} \leq \rho_{0}(x) \leq A_{1}\left|x-x_{0}\right|^{\sigma}, \text { for any } x \in \Omega, \\
u_{0} \in C^{1}(\bar{\Omega}), \quad\left(\rho_{0}^{\gamma-1+1 / 2 j}\right)_{x} \in L^{2 j}(\Omega), \rho_{0}^{-1+1 / 2 j}\left(\rho_{0}^{\alpha} u_{0 x}\right)_{x} \in L^{2 j}(\Omega), j=1, n,
\end{gathered}
$$

with $n \geq 2$ an integer; and in the case of continuous vacuum state of one piece initially on $\Omega^{0}=\left[x_{0}, x_{1}\right] \subset(0,1)$ in the initial data, we require

$$
\begin{cases}A_{0}\left(x_{0}-x\right)^{\sigma} \leq \rho_{0}(x) \leq A_{1}\left(x_{0}-x\right)^{\sigma}, & x \in\left[0, x_{0}\right), \\ \rho_{0}(x)=0, m_{0}(x)=\rho_{0} u_{0}(x)=0, & x \in\left[x_{0}, x_{1}\right], \\ B_{0}\left(x-x_{1}\right)^{\sigma} \leq \rho_{0}(x) \leq B_{1}\left(x-x_{1}\right)^{\sigma}, & x \in\left(x_{1}, 1\right]\end{cases}
$$

and

$$
\left\{\begin{array}{l}
\left(\rho_{0}^{\gamma-1+1 / 2 j}\right)_{x} \in L^{2 j}(\Omega), j=1, n, \quad u_{0} \in C^{1}\left(\bar{\Omega} \backslash \Omega^{0}\right), \\
\rho_{0}^{-1+1 / 2 j}\left(\rho_{0}^{\alpha} u_{0 x}\right)_{x} \in L^{2 j}\left(\Omega \backslash \Omega^{0}\right), j=1, n,
\end{array}\right.
$$

with $n \geq 2$ an integer. Here, $\sigma, A_{0}, A_{1}$, and $B_{0}, B_{1}$ are positive constants, and the power $\sigma \in\left(\sigma_{-}, \sigma_{+}\right)$with positive constants $\sigma_{ \pm}$given in (2.33) later. We also require that the initial data $\left(\rho_{0}, m_{0}\right)=\left(\rho_{0}, \rho_{0} u_{0}\right)$ given by (2.3) is consistent with boundary value for the Dirichlet boundary condition.

We have the following results on short time structure of global entropy weak solutions.

Theorem 2.2 (Short time structure of vacuum states) In addition to the assumptions of Theorem [2.1, assume further that

$$
\alpha>\frac{1}{2}, \quad \gamma>\max \{1, \alpha\}
$$

and that there is either one point vacuum state in initial data $\left(\rho_{0}, u_{0}\right)$ with (2.18)(2.19) satisfied or a piece of continuous vacuum states in initial data $\left(\rho_{0}, u_{0}\right)$ with (2.20) -(2.21) satisfied. Then, there exists a global entropy weak solution $(\rho, u)$ to the IBVP for the compressible Navier-Stokes equations (2.1) -(2.2) with initial data (2.3) and boundary value (2.6) or (2.7) in the sense of Definition 2.5.

Moreover, there is a short time $T_{*}>0$, so that the global entropy weak solution $(\rho, u)$ is uniqud and regular on the domain $\Omega \times\left[0, T_{*}\right]$, and the initial structure of vacuum states is maintained for $t \in\left[0, T_{*}\right]$ in the following sense:

For the case of one point vacuum state initially, (2.18), the solution $(\rho, u)$ is regular and unique on the domain $\bar{\Omega} \times\left[0, T_{*}\right]$,

$$
(\rho, u) \in C^{0}\left(\bar{\Omega} \times\left[0, T_{*}\right]\right), \quad u_{x} \in L^{\infty}\left(0, T_{*} ; C^{0}(\bar{\Omega})\right),
$$

\footnotetext{
${ }^{1}$ Here the uniqueness is specified for density $\rho$ and momentum $\rho u=\sqrt{\rho} \sqrt{\rho} u$ for continuous vacuum states of one piece.
} 


$$
\|u\|_{L^{\infty}\left(\bar{\Omega} \times\left[0, T_{*}\right]\right)}+\left\|u_{x}\right\|_{L^{\infty}\left(\left[0, T_{*}\right] ; C^{0}(\bar{\Omega})\right)} \leq C\left(T_{*}\right) .
$$

The one point vacuum state propagates along particle path, namely, there is one particle path $x=X_{0}(t):\left[0, T_{*}\right] \rightarrow \bar{\Omega}$ with $X_{0}(t) \in C\left(\left[0, T_{*}\right]\right)$ defined by

$$
\dot{X}_{0}(t)=u\left(X_{0}(t), t\right), \quad X_{0}(0)=x_{0} \in(0,1),
$$

so that

$$
a_{-}\left|x-X_{0}(t)\right|^{\sigma} \leq \rho(x, t) \leq a_{+}\left|x-X_{0}(t)\right|^{\sigma}
$$

for $(x, t) \in \Omega \times\left[0, T_{*}\right]$, where the two positive constants $a_{ \pm}$are independent of time $T_{*}$.

In the case of a piece of continuous vacuum states initially, (2.20), there are two particle pathes $x=X_{i}(t):\left[0, T_{*}\right] \rightarrow \bar{\Omega}$ with $X_{i}(t) \in C\left(\left[0, T_{*}\right]\right), i=0,1$ defined by

$$
\dot{X}_{i}(t)=u\left(X_{i}(t), t\right), \quad X_{i}(0)=x_{i} \in(0,1), i=0,1,
$$

so that it holds for some positive constants $a_{ \pm}, b_{ \pm}$independent of the time $T_{*}$,

$$
a_{-}\left(X_{0}(t)-x\right)^{\sigma} \leq \rho(x, t) \leq a_{+}\left(X_{0}(t)-x\right)^{\sigma},
$$

for $(x, t) \in\left[0, X_{0}(t)\right) \times\left[0, T_{*}\right]$, and

$$
b_{-}\left(x-X_{1}(t)\right)^{\sigma} \leq \rho(x, t) \leq b_{+}\left(x-X_{1}(t)\right)^{\sigma}
$$

for $(x, t) \in\left(X_{1}(t), 1\right] \times\left[0, T_{*}\right]$ respectively, and the interfaces separating the fluid and vacuum coincide with the particle pathes

$$
\rho(x, t)=0, \quad \rho u(x, t)=0, \quad(x, t) \in\left[X_{0}(t), X_{1}(t)\right] \times\left[0, T_{*}\right] .
$$

The solution $(\rho, u)$ is regular and unique up to the vacuum boundary

$$
\begin{gathered}
\rho \in C^{0}\left(\bar{\Omega} \times\left[0, T_{*}\right]\right), \quad u \in C^{0}\left(\bar{\Omega} \times\left[0, T_{*}\right] \backslash \Omega_{T_{*}}^{0}\right), \\
\|u\|_{L^{\infty}\left(\bar{\Omega} \times\left[0, T_{*}\right] \backslash \Omega_{T_{*}}^{0}\right)}+\left\|u_{x}\right\|_{L^{\infty}\left(\bar{\Omega} \times\left[0, T_{*}\right] \backslash \Omega_{T_{*}}^{0}\right)} \leq C\left(T_{*}\right) .
\end{gathered}
$$

where $\Omega_{T_{*}}^{0}=\left(X_{0}(t), X_{1}(t)\right) \times\left[0, T_{*}\right]$.

Remark 2.7 (1). The constant exponents $\sigma_{ \pm}$are defined as $\sigma_{ \pm}=\beta_{ \pm} /\left(1-\beta_{ \pm}\right)>0$ with $\beta_{ \pm}$determined by

$$
\beta_{-}=\max \left\{\frac{1}{2 \alpha}, \frac{1}{\gamma}\left(1-\frac{1}{2 n}\right)\right\}, \quad \beta_{+}=\min \left\{1, \frac{1}{\alpha}\left(1-\frac{1}{2 n}\right), \frac{1}{1+3 \alpha}\left(4-\frac{1}{n}\right)\right\},
$$

while the positive constants $a_{ \pm}$are independent of the time $T_{*}$.

(2). The regularity assumptions (2.18)-(2.19) are satisfied for the following initial data

$$
\rho_{0}(x)=\frac{1}{2}\left(A_{0}+A_{1}\right)\left(\left|x-x_{0}\right|^{2}\right)^{\left(\sigma_{-}+\sigma_{+}\right) / 4}, \quad u_{0}(x)=0, \quad x \in \Omega,
$$

and the regularity assumptions (2.20) -(2.21) are satisfied for the initial data

$$
\rho_{0}(x)= \begin{cases}\frac{1}{2}\left(A_{0}+A_{1}\right)\left(x_{0}-x\right)^{\left(\sigma_{-}+\sigma_{+}\right) / 2}, & x \in\left[0, x_{0}\right), \\ 0, & x \in\left(x_{0}, x_{1}\right), \quad u_{0}(x)=0, \quad x \in \Omega . \\ \frac{1}{2}\left(B_{0}+B_{1}\right)\left(x-x_{1}\right)^{\left(\sigma_{-}+\sigma_{+}\right) / 2}, & x \in\left(x_{1}, 1\right],\end{cases}
$$


Next, we prove that for any global entropy weak solution $(\rho, u)$ to the IBVP (2.1)(2.3) together with boundary condition (2.6) or (2.7) in the sense of Definition 2.5, even though in some cases that the vacuum states may exist for some finite time, for instance, in the cases as shown by Theorem 2.2 , any possible vacuum state has to vanish within finite time after which the density is always away from vacuum. Simultaneously, not only can the velocity field be defined in terms of the density and momentum, and the nonlinear diffusion is represented in terms of the density and velocity, but also the global entropy weak solution $(\rho, u)$ is shown to be a unique and strong solution after the vanishing of vacuum states. We have the following result.

Theorem 2.3 (Vanishing of vacuum states) Assume that

$$
\alpha>\frac{1}{2}, \quad \gamma \geq 1
$$

Let $(\rho, u)$ be any global entropy weak solution to the IBVP (2.1) -(2.2) with initial data (2.3) and boundary value (2.6) or (2.7) in the sense of Definition 2.5. Then, there exist some time $T_{0}>0$ (depending on initial data) and a constant $\rho_{-}$so that

$$
\inf _{x \in \bar{\Omega}} \rho(x, t) \geq \rho_{-}>0, \quad t \geq T_{0},
$$

and the global entropy weak solution $(\rho, u)$ becomes a unique strong solution $(\rho, u)$ for $t \geq T_{0}$ and satisfies

$$
\left\{\begin{array}{l}
\rho \in L^{\infty}\left(T_{0}, t ; H^{1}(\Omega)\right), \quad \rho_{t} \in L^{\infty}\left(T_{0}, t ; L^{2}(\Omega)\right), \\
u \in H^{1}\left(T_{0}, t ; L^{2}(\Omega)\right) \cap L^{2}\left(T_{0}, t ; H^{2}(\Omega)\right),
\end{array}\right.
$$

with velocity $u$ and nonlinear diffusion term given by

$$
u \triangleq \frac{\sqrt{\rho} u}{\sqrt{\rho}}, \quad\left(\rho^{\alpha} u_{x}\right)_{x}=\Lambda_{x}
$$

respectively. In addition, for

$$
u_{s} \triangleq \begin{cases}0 & \text { for the Dirichlet case } \\ \frac{1}{\overline{\rho_{0}}} \int_{\Omega} m_{0} d x & \text { for the periodic case }\end{cases}
$$

there exist two positive constants $\mu_{0}, c_{0}$ both depending on initial data $\left(\rho_{0}, m_{0}\right)$ and $\rho_{-}$, such that

$$
\left\|\left(\rho-\overline{\rho_{0}}, u-u_{s}\right)(\cdot, t)\right\|_{L^{2}(\Omega)} \leq c_{0} e^{-\mu_{0}\left(t-T_{0}\right)}, \quad t>T_{0},
$$

where and what follows $\bar{f}$ denotes the average of $f$ over the bounded domain $\Omega$, i.e.,

$$
\bar{f}=\frac{1}{|\Omega|} \int_{\Omega} f(x) d x=\int_{\Omega} f(x) d x .
$$


Remark 2.8 (1). The Theorem 2.3 shows that any possible vacuum states must vanish in finite time. This theory applies to the compressible shallow water equation (1.2).

(2). It is easy to verify (see the proof of Proposition 5.1) that the phenomena of vacuum vanishing (2.35) in finite time actually happens for any global weak solution $(\rho, u)$ to the IBVP (2.1) - (2.3) with boundary condition (2.6) or (2.7) in the sense of Definition 2.3 satisfying the following entropy inequality

$$
\begin{gathered}
\sup _{0 \leq t \leq T} \int_{\Omega}\left(|\sqrt{\rho} u|^{2}+\left|\left(\rho^{\alpha-1 / 2}\right)_{x}\right|^{2}+\pi(\rho)\right)(x, t) d x+\int_{0}^{T}\left\|\left(\rho^{(\gamma+\alpha-1) / 2}\right)_{x}\right\|_{L^{2}}^{2} d t \\
\leq C_{0} \int_{\Omega}\left(\frac{\left|m_{0}\right|^{2}}{\rho_{0}}+\left|\left(\rho_{0}^{\alpha-1 / 2}\right)_{x}\right|^{2}+\pi_{+}\left(\rho_{0}\right)\right)(x) d x
\end{gathered}
$$

with $C_{0}$ independent of $T$.

Finally, for any global entropy weak solution $(\rho, u)$ to the IBVP (2.1)-(2.3) together with boundary condition (2.6) or (2.7) in the sense of Definition 2.5, the density is continuous, i.e., $\rho \in C(\bar{\Omega} \times[0, T])$ for any $T>0$, due to (2.8) and (2.9). Thus, the continuity of $\rho$ and Theorem 2.3 imply that if the density contains vacuum states at least at one point, then there exists some critical time $T_{1} \in\left[0, T_{0}\right)$ with $T_{0}>0$ given by (2.35) and a nonempty subset $\Omega^{0} \subset \bar{\Omega}$ such that

$$
\begin{cases}\rho\left(x, T_{1}\right)=0, & \forall x \in \Omega^{0} \\ \rho\left(x, T_{1}\right)>0, & \forall x \in \bar{\Omega} \backslash \Omega^{0}, \\ \rho(x, t)>0, & \forall(x, t) \in \bar{\Omega} \times\left(T_{1}, T_{0}\right] .\end{cases}
$$

It follows from (2.36) easily that for any $\delta>0$, it holds

$$
\int_{T_{1}+\delta}^{T_{0}}\left\|u_{x}\right\|_{L^{\infty}} d s<\infty .
$$

Under the condition that vacuum states appear, we shall prove that the spatial derivative of velocity (if regular enough and definable) blows up in finite time as the vacuum states vanish, even if the solution is regular enough for short time so that the velocity field and its derivatives are bounded as shown by Theorem 2.2 .

Theorem 2.4 (Finite time blow-up) Let $(\rho, u)$ be any global entropy weak solution, which contains vacuum states at least at one point for some finite time, to the IBVP for the compressible Navier-Stokes equations (2.1) -(2.3) with boundary condition (2.6) or (2.7) in the sense of Definition 2.5. Let $T_{0}>0$ and $T_{1} \in\left[0, T_{0}\right)$ be the time such that (2.35) and 2.40) holds respectively.

Then, the solution $(\rho, u)$ blows up as vacuum states vanish. Namely, for $T_{1}$ satisfying (2.40) and for given any fixed $\eta>0$, it holds

$$
\lim _{t \rightarrow T_{1}^{+}} \int_{t}^{T_{1}+\eta}\left\|u_{x}\right\|_{L^{\infty}} d s=\infty .
$$


On the other hand, if there exists some $T_{2} \in\left(0, T_{0}\right)$ such that the weak solution $(\rho, u)$ satisfies

$$
\|u\|_{L^{1}\left(0, T_{2} ; W^{1, \infty}(\Omega)\right)}<\infty
$$

then, there is a time $T_{3} \in\left[T_{2}, T_{0}\right)$ so that the blowup phenomena happens for $(\rho, u)$, i.e.,

$$
\lim _{t \rightarrow T_{3}^{-}} \int_{0}^{t}\left\|u_{x}\right\|_{L^{\infty}} d s=\infty .
$$

Remark 2.9 Theorem 2.4 implies that for any global entropy weak solution $(\rho, u)$ to the IBVP for the compressible Navier-Stokes equations (2.1)-(2.2) with initial data (2.3) and boundary value (2.6) or (2.7) in the sense of Definition 2.5, which contains vacuum states at least at one point initially, the finite time blowup phenomena (2.42) happens for such solution $(\rho, u)$.

Remark 2.10 Theorems 2.1 2.4 provide a complete dynamical description on the vanishing of vacuum states and blow-up phenomena for the global entropy weak solutions to the compressible Navier-Stokes equations with density-dependent viscosity. That is, a global entropy weak solution exists for general large initial data with finite entropy. For short time, such weak solution is unique and regular with well-defined velocity field subject to additional initial regularity, and any existing vacuum state is maintained with the same interface structure as initial. Then, within finite time the vacuum states vanish definitely and the velocity blows up (even if it is regular enough and definable along the interfaces). After the vanishing of vacuum states, the global entropy weak solution becomes a strong one and tends to the non-vacuum equilibrium state exponentially in time. This dynamical phenomena is quite similar to those well-known for the 3-D incompressible Navier-Stokes equations. However, before the time of vacuum-vanishing, the uniqueness of the global entropy weak solution to the compressible Navier-Stokes equations with density-dependent viscosity subject to the initial data is not known yet.

Remark 2.11 All theories established in Theorems 2.1 2.4 fit the shallow water equation (1.2). We believe that such phenomena described by Theorems 2.12.4 are also observed for other compressible fluids with density-dependent viscosity, such as NavierStokes equations with capillarity and/or drag friction, Navier-Stokes-Poisson system, etc.

Remark 2.12 It is interesting to investigate the (global) dynamics of (one-dimensional) interface connecting vacuum from initial time until the vanishing of vacuum in order to investigate the dynamics of interface and the vanishing of vacuum state and to verify the formation of singularity for general case.

Remark 2.13 (1). Another interesting problem is whether the phenomena of the vanishing of vacuum states and blow-up happens for multi-dimensional compressible isentropic Navier-Stokes system with density-dependent viscosity, especially on spatial bounded domain with the Dirichlet boundary condition (2.6). 
(2). It is also interesting to study whether any vacuum states shall vanish within finite time and blow-up phenomena happens for the multi-dimensional full Navier-Stokes system with density-dependent viscosity. It is not obvious yet (although we expect) since it is not clearly understood yet how the dynamics of temperature and heat-conduction shall affect the global existence of weak solutions and the evolution of vacuum states, especially in the case of spherical symmetry under the Dirichlet boundary condition (2.6). This is under further investigation [26].

\section{Global existence of entropy weak solutions}

In this section, we will establish the existence of global entropy weak solutions to the IBVP for the compressible Navier-Stokes equations (2.1)-(2.3) together with (2.6) or (2.7). Since the compactness arguments are straightforward in the framework of Mellet-Vasseur [38], we only need to construct a sequence of approximate solutions by using some ideas developed by Jiang-Xin-Zhang in [23] for one-dimensional compressible Navier-Stokes equations with free boundary, to establish some uniform a priori estimates, in particular, the lower and upper bounds of the density for the approximate solution sequence with the help of BD entropy, and to justify the boundary condition (2.16) for (limiting) weak solution for the Dirichlet case as follows.

Outline of proof of Theorem 2.1. Step 1. Construction of smooth approximate solutions. Let us consider the following approximate compressible Navier-Stokes equations inspired by Jiang-Xin-Zhang in [23]

$$
\begin{gathered}
\rho_{\varepsilon t}+\left(\rho_{\varepsilon} u_{\varepsilon}\right)_{x}=0 \\
\left(\rho_{\varepsilon} u_{\varepsilon}\right)_{t}+\left(\rho_{\varepsilon} u_{\varepsilon}^{2}+p\left(\rho_{\varepsilon}\right)\right)_{x}-\left(\mu_{\varepsilon}\left(\rho_{\varepsilon}\right) u_{\varepsilon x}\right)_{x}=0 \\
\left(\rho_{\varepsilon}, \rho_{\varepsilon} u_{\varepsilon}\right)(x, 0)=\left(\rho_{0 \varepsilon}, m_{0 \varepsilon}\right)(x)
\end{gathered}
$$

with one of the following boundary conditions

$$
u_{\varepsilon}(0, t)=u_{\varepsilon}(1, t)=0,
$$

or

$$
\rho_{\varepsilon}, u_{\varepsilon} \text { are periodic in } x \text { of period } 1 .
$$

The viscosity $\mu_{\varepsilon}$ is given by

$$
\mu_{\varepsilon}(\rho)=\rho^{\alpha}+\varepsilon \rho^{\theta}, \quad \varepsilon>0, \quad \theta \in(0,1 / 2),
$$

where we remark (see Remark 3.1 below) that although the modified viscosities (3.6) are limited to the case $\theta \in(0,1 / 2)$, the methods adapted here to construct approximate solutions also can be applied to the case $\theta \in(0,1)$ and $\gamma>\theta$ after some modification, the interesting reader can refer to $[16,23]$ and references therein, we omit the details here. The initial data $\rho_{0 \varepsilon}, m_{0 \varepsilon} \in C^{\infty}(\bar{\Omega})$ satisfies

$$
\left\{\begin{array}{l}
\rho_{0 \varepsilon} \rightarrow \rho_{0} \text { in } L^{1}(\Omega), \rho_{0 \varepsilon}^{\alpha-1 / 2} \rightarrow \rho_{0}^{\alpha-1 / 2} \text { in } H^{1}(\Omega), \\
\left(m_{0 \varepsilon}\right)^{2}\left(\rho_{0 \varepsilon}\right)^{-1} \rightarrow m_{0}^{2} \rho_{0}^{-1},\left|m_{0 \varepsilon}\right|^{2+\nu}\left(\rho_{0 \varepsilon}\right)^{-1-\nu} \rightarrow\left|m_{0}\right|^{2+\nu} \rho_{0}^{-1-\nu} \text { in } L^{1}(\Omega)
\end{array}\right.
$$


as $\varepsilon \rightarrow 0^{+}$, and

$$
\rho_{0 \varepsilon} \geq c_{0} \varepsilon^{1 /(2 \alpha-2 \theta)}
$$

for some $c_{0}$ independent of $\varepsilon$. By (3.7) we can assume without the loss of generality that

$$
\int_{\Omega} \rho_{0 \varepsilon} d x=1
$$

By the standard arguments (see [23] for reference), after applying the classical theory of parabolic and hyperbolic equations, one can obtain that there exists some $T_{*}>0$ such that the approximate problem (3.1)-(3.3) together with the boundary condition (3.4) or (3.5) has a unique smooth solution $\left(\rho_{\varepsilon}, u_{\varepsilon}\right)$ on $\left[0, T_{*}\right]$ with the density away from vacuum, i.e.,

$$
\rho_{\varepsilon}(x, t)>0, \text { for all }(x, t) \in \bar{\Omega} \times\left[0, T_{*}\right] .
$$

Step 2. The a-priori estimates. To extend the local solution globally in time, one needs to control the lower and upper bounds of the density and get some a-priori estimates. As mentioned in the introduction, this relies on the BD entropy inequality developed by Bresch and Desjardins (see $[2,3,6,7]$ for instance). In the Eulerian coordinates, the BD entropy for the approximate solutions $\left(\rho_{\varepsilon}, u_{\varepsilon}\right)$ of the IBVP problem for the system (3.1)-(3.1) reads (see $[2,3,6,7]$ for instance) as

$$
\begin{aligned}
\int_{\Omega}\left(\mid \sqrt{\rho_{\varepsilon}}\right. & \left.\left.u_{\varepsilon}\right|^{2}+\left|\frac{\mu\left(\rho_{\varepsilon}\right)_{x}}{\sqrt{\rho_{\varepsilon}}}\right|^{2}+\left|\frac{\varepsilon\left(\left(\rho_{\varepsilon}\right)^{\theta}\right) x}{\sqrt{\rho_{\varepsilon}}}\right|^{2}+\pi\left(\rho_{\varepsilon}\right)\right)(x, t) d x \\
& \quad+\int_{0}^{t} \int_{\Omega}\left(\mu_{\varepsilon}\left(\rho_{\varepsilon}\right)\left|u_{\varepsilon x}\right|^{2}+\frac{p^{\prime}\left(\rho_{\varepsilon}\right) \mu_{\varepsilon}^{\prime}\left(\rho_{\varepsilon}\right)}{\rho_{\varepsilon}}\left|\rho_{\varepsilon x}\right|^{2}\right)(x, s) d x d s \leq C, t>0
\end{aligned}
$$

with positive constant $C$ independent of $t$ and $\varepsilon$. Indeed, multiplying (3.2) by $u_{\varepsilon}$, integrating by parts on $[0,1]$, and using (3.1), we get the classical entropy estimate

$$
\int_{\Omega}\left(\left|\sqrt{\rho_{\varepsilon}} u_{\varepsilon}\right|^{2}+\pi\left(\rho_{\varepsilon}\right)\right)(x, t) d x+\int_{0}^{t} \int_{\Omega} \mu_{\varepsilon}\left(\rho_{\varepsilon}\right)\left|u_{\varepsilon x}\right|^{2} d x d s \leq C, t>0
$$

with positive constant $C$ independent of $t$ and $\varepsilon$.

Multiplying the transport equation (3.1) by $\varphi_{\varepsilon}^{\prime}\left(\rho_{\varepsilon}\right)$ with $\rho \varphi_{\varepsilon}^{\prime}(\rho)=\mu_{\varepsilon}(\rho)$, i.e., $\varphi_{\varepsilon}(\rho)=$ $\frac{1}{\alpha} \rho^{\alpha}+\frac{\varepsilon}{\theta} \rho^{\theta}$ as in [8], we get

$$
\varphi_{\varepsilon}\left(\rho_{\varepsilon}\right)_{t}+\varphi_{\varepsilon}\left(\rho_{\varepsilon}\right)_{x} u_{\varepsilon}+\varphi_{\varepsilon}^{\prime}\left(\rho_{\varepsilon}\right) \rho_{\varepsilon} u_{\varepsilon x}=0 .
$$

Differentiating above equation with respect to $x$ and denoting $v_{\varepsilon}=\varphi_{\varepsilon}\left(\rho_{\varepsilon}\right)_{x} / \rho_{\varepsilon}$, we have

$$
\left(\rho_{\varepsilon} v_{\varepsilon}\right)_{t}+\left(\rho_{\varepsilon} v_{\varepsilon} u_{\varepsilon}\right)_{x}+\left(\mu_{\varepsilon}\left(\rho_{\varepsilon}\right) u_{\varepsilon x}\right)_{x}=0 .
$$

Summing the equations (3.14) and (3.1) together, multiplying by $u_{\varepsilon}+v_{\varepsilon}$ and integrating by parts on $[0,1]$, we have after a straightforward computation that

$$
\begin{aligned}
\int_{\Omega}\left(\left|\sqrt{\rho_{\varepsilon}} u_{\varepsilon}\right|^{2}+\left|\frac{\varphi_{\varepsilon}\left(\rho_{\varepsilon}\right)_{x}}{\sqrt{\rho_{\varepsilon}}}\right|^{2}+\pi\left(\rho_{\varepsilon}\right)\right)(x, t) d x \\
\quad+\int_{0}^{t} \int_{\Omega}\left(\mu_{\varepsilon}\left(\rho_{\varepsilon}\right)\left|u_{\varepsilon x}\right|^{2}+\frac{p^{\prime}\left(\rho_{\varepsilon}\right) \varphi_{\varepsilon}^{\prime}\left(\rho_{\varepsilon}\right)}{\rho_{\varepsilon}}\left|\rho_{\varepsilon x}\right|^{2}\right)(x, s) d x d s \leq C, t>0
\end{aligned}
$$


with positive constant $C$ independent of $t$ and $\varepsilon$. This together with (3.12)

$$
\begin{aligned}
\left(\rho_{\varepsilon}^{2 \alpha-2}+\varepsilon^{2} \rho_{\varepsilon}^{2 \theta-2}\right)\left(\rho_{\varepsilon x}\right)^{2} & \leq\left(\varphi_{\varepsilon}\left(\rho_{\varepsilon}\right)_{x}\right)^{2}=\left(\rho_{\varepsilon}^{2 \alpha-2}+2 \varepsilon \rho_{\varepsilon}^{\alpha+\theta-2}+\varepsilon^{2} \rho_{\varepsilon}^{2 \theta-2}\right)\left(\rho_{\varepsilon x}\right)^{2} \\
& \leq 2\left(\rho_{\varepsilon}^{2 \alpha-2}+\varepsilon^{2} \rho_{\varepsilon}^{2 \theta-2}\right)\left(\rho_{\varepsilon x}\right)^{2}
\end{aligned}
$$

gives rise to the BD entropy (3.11).

In order to derive the lower bound and upper bounds of density for the approximate solution $\left(\rho_{\varepsilon}, u_{\varepsilon}\right)$, it is convenient to make use of the Lagrangian coordinates $(y, t)$ with

$$
y=\int_{0}^{x} \rho_{\varepsilon}(z, t) d z, t=t
$$

because the approximate solution $\left(\rho_{\varepsilon}, u_{\varepsilon}\right)$ is away from vacuum at least for short time due to (3.10) and fluid density transports along particle path. In Lagrangian coordinates, we have the equivalent system for $\left(\rho_{\varepsilon}, u_{\varepsilon}\right)$ as follows

$$
\begin{gathered}
\rho_{\varepsilon t}+\rho_{\varepsilon}^{2} u_{\varepsilon y}=0, \\
u_{\varepsilon t}+\left(p\left(\rho_{\varepsilon}\right)\right)_{y}-\left(\rho_{\varepsilon} \mu_{\varepsilon}\left(\rho_{\varepsilon}\right) u_{\varepsilon y}\right)_{y}=0, \\
\left(\rho_{\varepsilon}, u_{\varepsilon}\right)(y, 0)=\left(\rho_{0 \varepsilon}, m_{0 \varepsilon} \rho_{0 \varepsilon}^{-1}\right)(y),
\end{gathered}
$$

together with the boundary conditions similar to (3.4) or (3.5), where $(y, t) \in \Omega_{L} \times$ $\left(0, T_{*}\right]$ and

$$
\Omega_{L} \triangleq\left(0, L_{\varepsilon}\right), \text { with } L_{\varepsilon}=\int_{\Omega} \rho_{0 \varepsilon}(x) d x=1
$$

due to (3.9).

Correspondingly, the BD entropy equality (3.11) becomes in Lagrangian coordinates as follows

$$
\begin{aligned}
\sup _{0 \leq t \leq T_{*}} & E_{\varepsilon}(t)+\int_{0}^{T_{*}} \int_{\Omega_{L}} \rho_{\varepsilon} \mu_{\varepsilon}\left(\rho_{\varepsilon}\right)\left(u_{\varepsilon y}\right)^{2} d y d t \\
& +\int_{0}^{T_{*}} \int_{\Omega_{L}}\left(\rho_{\varepsilon}^{\gamma+\alpha-2}+\varepsilon \rho_{\varepsilon}^{\gamma+\theta-2}\right)\left(\rho_{\varepsilon y}\right)^{2} d y d t \leq C_{0} E_{\varepsilon}(0)
\end{aligned}
$$

with $C_{0}$ independent of $T_{*}$ and $\varepsilon>0$, and $E_{\varepsilon}(t)$ defined as

$$
E_{\varepsilon}(t) \triangleq \int_{\Omega_{L}}\left(u_{\varepsilon}^{2}+\left(\left(\rho_{\varepsilon}^{\alpha}\right)_{y}\right)^{2}+\varepsilon^{2}\left(\left(\rho_{\varepsilon}^{\theta}\right)_{y}\right)^{2}+\rho_{\varepsilon}^{-1} \pi\left(\rho_{\varepsilon}\right)\right) d y .
$$

Since

$$
\int_{\Omega_{L}} \rho_{\varepsilon}^{-1} d y=\int_{\Omega} d x=|\Omega|=1
$$

it follows from the continuity of $\rho_{\varepsilon}$ that there exists some $y_{0}(t) \in \Omega_{L}$ such that

$$
\rho_{\varepsilon}\left(y_{0}(t), t\right)=\left|\Omega_{L}\right| /|\Omega|=1
$$


Hence, it implies from (3.19) that

$$
\begin{aligned}
\rho_{\varepsilon}^{\alpha}(y, t) & =\rho_{\varepsilon}^{\alpha}\left(y_{0}(t), t\right)+\int_{y_{0}(t)}^{y}\left(\rho_{\varepsilon}^{\alpha}\right)_{y} d y \\
& \leq\left(\left|\Omega_{L}\right||\Omega|^{-1}\right)^{\alpha}+C_{0} E_{\varepsilon}(0)+\left|\Omega_{L}\right| .
\end{aligned}
$$

This yields the uniform upper bound (w.r.t. time $t \in\left[0, T_{*}\right]$ ) for the density.

To obtain the lower bound for the density $\rho_{\varepsilon}$, we employ the idea in [23]. That is, we consider the upper bound for $v_{\varepsilon}=\rho_{\varepsilon}^{-1}$, which can be estimated by (3.19) and (3.20) as follow:

$$
\begin{aligned}
v_{\varepsilon}(y, t) & \leq \int_{\Omega_{L}} v_{\varepsilon}(y, t) d y+\int_{\Omega_{L}}\left(v_{\varepsilon}\right)^{2}\left|\rho_{\varepsilon y}\right| d y \\
& \leq 1+C \max _{y \in \bar{\Omega}_{L}}\left(v_{\varepsilon}\right)^{\theta+1 / 2}\left\|\left(\rho_{\varepsilon}^{\theta}\right)_{y}\right\|_{L^{2}(\Omega)}\left(\int_{\Omega_{L}} v_{\varepsilon}(y, t) d y\right)^{1 / 2} \\
& \leq 1+\frac{1}{2} \max _{y \in \bar{\Omega}_{L}} v_{\varepsilon}+C\left(\varepsilon^{-2} E_{\varepsilon}(0)\right)^{1 /(1-2 \theta)} .
\end{aligned}
$$

This shows that for all $(y, t) \in \bar{\Omega}_{L} \times\left[0, T_{*}\right]$,

$$
\rho_{\varepsilon}(y, t) \geq \frac{\varepsilon^{2 /(1-2 \theta)}}{2 \varepsilon^{2 /(1-2 \theta)}+C\left(E_{\varepsilon}(0)\right)^{1 /(1-2 \theta)}} .
$$

Thus, we have obtained the uniform upper bound (3.21) and the lower bound (3.23) (w.r.t. time $t \in\left[0, T_{*}\right]$ ) for density $\rho_{\varepsilon}$. Using these uniform bounds, one can extend the local smooth solution globally in time by standard arguments (see [23] for details). Moreover, the total mass of the approximate solutions is conserved due to the boundary conditions (3.4)

$$
\int_{\Omega} \rho_{\varepsilon}(x, t) d x=\int_{\Omega} \rho_{0 \varepsilon}(x) d x, \quad \forall t>0
$$

Remark 3.1 Although the estimates (3.22) and (3.23) are established for the approximate solutions in the case that the modified viscosities (3.6) are limited to the case $\theta \in(0,1 / 2)$, the methods adapted here can be applied to establish the lower bounds like (3.23) in the case $\theta \in(0,1)$ and $\gamma>\theta$. Indeed, one can also establish the following estimates, similar to [16,23], for the approximate solutions $\left(\rho, u_{\varepsilon}\right)$ as

$$
\sup _{t \in[0, T]} \int\left(\rho^{\theta}\right)_{x}^{2 n}(x, t) d x \leq C(T)
$$

for any integer $n \geq 1$ and $C(T)$ a constant independent of $\varepsilon, \theta$. Thus, we can verify similar to (3.22) that

$$
v_{\varepsilon} \leq 1+C \int v_{\varepsilon}^{1+\theta}\left|\left(\rho^{\theta}\right)_{x}\right| d x \leq 1+C(T)\left(\int v_{\varepsilon}^{q(\theta+1)} d y\right)^{1 / q} \leq C+C(T) v_{\varepsilon}^{(q(\theta+1)-1) / q}
$$


with $q=2 n /(2 n-1)$. This together with Young's inequality implies the positive lower bound of density for integer $n$ sufficient large but fixed so that $\theta<\frac{2 n-1}{2 n}$ so long as $\theta \in(0,1)$.

In addition to (3.11), we show $\rho_{\varepsilon}^{\eta} u_{\varepsilon}$ is actually bounded in $L^{\infty}\left(0, T ; L^{2+\nu}(\Omega)\right)$ for some $\eta \geq 1 /(2+\nu)$ with $\nu>0$ given by

$$
\begin{cases}\nu>0 \text { arbitrary } & \text { if } 2 \gamma-\alpha \geq 1 \\ \nu \in(0,2(2 \gamma-\alpha) /(1+\alpha-2 \gamma)] & \text { if } 2 \gamma-\alpha \in(0,1) .\end{cases}
$$

Note here that for $\alpha \in(1 / 2,3 / 2)$ the constant $\nu$ defined by (2.17) actually satisfies (3.25). It follows from (3.11) that

$$
\sup _{t \geq 0}\left\|\left(\rho_{\varepsilon}^{\alpha-1 / 2}\right)_{x}\right\|_{L^{2}(\Omega)} \leq \bar{C}
$$

which together with (3.24) and the mean value theorem gives rise to

$$
\sup _{t \geq 0}\left\|\rho_{\varepsilon}\right\|_{L^{\infty}}^{\alpha-1 / 2} \leq{\overline{\rho_{0 \varepsilon}}}^{\alpha-1 / 2}+\left\|\left(\rho_{\varepsilon}^{\alpha-1 / 2}\right)_{x}\right\|_{L^{2}(\Omega)} \leq \bar{C}
$$

where $\bar{C}$ denotes some generic positive constant depending on $E_{\varepsilon}(0)$ but independent of $T$. With the help of (3.27), we can make use of the idea due to [38] to derive the following a-priori estimate

$$
\sup _{0 \leq t \leq T} \int_{\Omega} \rho_{\varepsilon}\left|u_{\varepsilon}\right|^{2+\nu} d x \leq C
$$

which together with (3.27) implies for $\eta(2+\nu) \geq 1$ that

$$
\int_{\Omega}\left(\rho_{\varepsilon}^{\eta}\left|u_{\varepsilon}\right|\right)^{2+\nu} d x=\int_{\Omega} \rho_{\varepsilon}^{\eta(2+\nu)}\left|u_{\varepsilon}\right|^{2+\nu} d x \leq C \sup _{0 \leq t \leq T} \int_{\Omega} \rho_{\varepsilon}\left|u_{\varepsilon}\right|^{2+\nu} d x \leq C,
$$

and, in particular, for $\eta=1 / 2$ that

$$
\sup _{0 \leq t \leq T} \int_{\Omega}\left(\sqrt{\rho_{\varepsilon}}\left|u_{\varepsilon}\right|\right)^{2+\nu} d x \leq C
$$

with $C$ one generic positive constant depending on both $T$ and $E_{\varepsilon}(0)$.

Thus, we can follow the compactness arguments in [38] to prove the convergence of the approximate solution $\left(\rho_{\varepsilon}, u_{\varepsilon}\right)$ to some expected weak solution $(\rho, u)$ as $\varepsilon \rightarrow 0+$. Namely, it holds

$$
\begin{gathered}
\rho_{\varepsilon} \longrightarrow \rho \quad \text { in } C([0, T] \times \bar{\Omega}), \\
\left(\rho_{\varepsilon}^{\alpha-1 / 2}\right)_{x} \rightarrow\left(\rho^{\alpha-1 / 2}\right)_{x} \quad \text { weakly in } L^{2}(\Omega \times(0, T)), \\
\sqrt{\rho_{\varepsilon}} u_{\varepsilon} \longrightarrow \sqrt{\rho} u, \quad \rho_{\varepsilon}^{\alpha} u_{\varepsilon} \longrightarrow \rho^{\alpha} u \quad \text { in } L^{2+\nu / 2}(\Omega \times(0, T)),
\end{gathered}
$$


and there exists some function $\Lambda \in L^{2}(\Omega \times(0, T))$ such that

$$
\rho_{\varepsilon}^{\alpha} u_{\varepsilon x} \rightarrow \Lambda \quad \text { weakly in } L^{2}(\Omega \times(0, T)) \text {, as } \varepsilon \rightarrow 0 .
$$

Step 3. Justification of the Dirichlet boundary condition (2.16) for $\alpha \in(1 / 2,3 / 2)$. For $\alpha \in(1 / 2,1]$, it follows from (3.11) and (3.29) that

$$
\begin{aligned}
& \left\|\left(\rho_{\varepsilon} u_{\varepsilon}\right)_{x}\right\|_{L^{2}\left(0, T ; L^{(4+2 \nu) /(4+\nu)}(\Omega)\right)} \\
& \quad \leq C\left\|\rho_{\varepsilon}^{\alpha / 2} u_{\varepsilon x}\right\|_{L^{2}\left(0, T ; L^{2}(\Omega)\right)}+C\left\|\left(\rho_{\varepsilon}^{\alpha-1 / 2}\right)_{x} \rho_{\varepsilon}^{1 / 2} u_{\varepsilon}\right\|_{L^{2}\left(0, T ; L^{(4+2 \nu) /(4+\nu)}(\Omega)\right)} \\
& \quad \leq C+C\left\|\left(\rho_{\varepsilon}^{\alpha-1 / 2}\right)_{x}\right\|_{L^{\infty}\left(0, T ; L^{2}(\Omega)\right)} \cdot\left\|\rho_{\varepsilon}^{1 / 2} u_{\varepsilon}\right\|_{L^{\infty}\left(0, T ; L^{2+\nu}(\Omega)\right)} \\
& \quad \leq C .
\end{aligned}
$$

For $\alpha \in(1,3 / 2)$, some additional estimates are needed here. First, for $\gamma \geq(1+\alpha) / 2$, it is easy to check that for $\nu$ defined by (2.17) that $(3 / 2-\alpha) /(2+\nu)>1$. Hence, for $\beta=(4+2 \nu) /(4+\nu)$ one deduces from (3.29) and (3.27) that

$$
\begin{aligned}
& \left\|\left(\rho_{\varepsilon} u_{\varepsilon}\right)_{x}\right\|_{L^{2}\left(0, T ; L^{\beta}(\Omega)\right)} \\
& \quad \leq C\left\|\rho_{\varepsilon}^{\alpha / 2} u_{\varepsilon x}\right\|_{L^{2}\left(0, T ; L^{2}(\Omega)\right)}+C\left\|\left(\rho_{\varepsilon}^{\alpha-1 / 2}\right)_{x} \rho_{\varepsilon}^{-\alpha+3 / 2} u_{\varepsilon}\right\|_{L^{2}\left(0, T ; L^{\beta}(\Omega)\right)} \\
& \quad \leq C+C\left\|\left(\rho_{\varepsilon}^{\alpha-1 / 2}\right)_{x}\right\|_{L^{\infty}\left(0, T ; L^{2}(\Omega)\right)}\left\|\rho_{\varepsilon}^{-\alpha+3 / 2} u_{\varepsilon}\right\|_{L^{\infty}\left(0, T ; L^{2+\nu}(\Omega)\right)} \\
& \quad \leq C .
\end{aligned}
$$

In the case that $1 \leq \gamma<(1+\alpha) / 2$, (2.17) yields that $(3-\alpha-\gamma)(2+\nu) \geq 2$. Hence, one derives from (3.29) and (3.11) that

$$
\begin{aligned}
& \left\|\left(\rho_{\varepsilon} u_{\varepsilon}\right)_{x}\right\|_{L^{2}\left(0, T ; L^{\beta}(\Omega)\right)} \\
& \quad \leq C\left\|\rho_{\varepsilon}^{\alpha / 2} u_{\varepsilon x}\right\|_{L^{2}\left(0, T ; L^{2}(\Omega)\right)}+C\left\|\left(\rho_{\varepsilon}^{(\gamma+\alpha-1) / 2}\right)_{x} \rho_{\varepsilon}^{(3-\alpha-\gamma) / 2} u_{\varepsilon}\right\|_{L^{2}\left(0, T ; L^{\beta}(\Omega)\right)} \\
& \quad \leq C+C\left\|\left(\rho_{\varepsilon}^{(\gamma+\alpha-1) / 2}\right)_{x}\right\|_{L^{2}\left(0, T ; L^{2}(\Omega)\right)}\left\|\rho_{\varepsilon}^{(3-\alpha-\gamma) / 2} u_{\varepsilon}\right\|_{L^{\infty}\left(0, T ; L^{2+\nu}(\Omega)\right)} \\
& \quad \leq C .
\end{aligned}
$$

Thus, the estimates (3.4), (3.36) and (3.37) imply that (2.16) still holds for $\alpha \in(1,3 / 2)$. The proof of the Theorem 2.1 on the existence of global entropy weak solution $(\rho, u)$ is completed.

\section{Dynamics of vacuum states for short time}

\subsection{Short time structure of vacuum states}

We prove the Theorem 2.2 in this subsection in order to study the short time structure of vacuum states for global entropy weak solutions. To this end, it is sufficient to show that there is a unique entropy weak solution in short time for the compressible Navier-Stokes equations (2.1)-(2.2) with initial data (2.3) and boundary value (2.6) or (2.7) under the assumptions of Theorem 2.2 as follows. 
Proposition 4.1 (Vacuum states for short time) Under the assumptions of Theorem 2.2. there is a short time $T_{*}^{\prime}>0$ so that the unique entropy weak solution $(\tilde{\rho}, \tilde{u})$ in the sense of Definition 2.5 of the IBVP problem for the compressible Navier-Stokes equations (2.1) -(2.2) with initial data (2.3) and boundary condition (2.6) or (2.7) exists on the domain $\Omega \times\left[0, T_{*}^{\prime}\right]$. The initial vacuum state (2.19) or (2.21) is also propagated for the short time $t \in\left[0, T_{*}^{\prime}\right]$, more precisely, the properties (2.23)-(2.26) or (2.27)(2.32) hold respectively for $(\tilde{\rho}, \tilde{u})$. In addition, it holds that

$$
\left\|\left(\tilde{\rho}^{\alpha-1 / 2}\right)_{x}\right\|_{L^{\infty}\left(0, T_{*}^{\prime} ; L^{2}(\Omega)\right)}+\left\|\left((\tilde{\rho} \tilde{u})^{2} / \tilde{\rho},(\tilde{\rho} \tilde{u})^{2+\nu} / \tilde{\rho}^{1+\nu}\right)\right\|_{L^{\infty}\left(0, T_{*}^{\prime} ; L^{1}(\Omega)\right)} \leq C\left(T_{*}^{\prime}\right) .
$$

Proof: The proof of Proposition 4.1 will be completed in subsections 4.24 .3 later.

Proof of Theorem 2.2. This is a consequence of the Proposition 4.1 and Theorem 2.1. In fact, the Proposition 4.1 shows not only that there exists a unique entropy weak solution $(\tilde{\rho}, \tilde{u})$ on the domain $\Omega \times\left(0, T_{*}^{\prime}\right)$ in the sense of Definition 2.5 to the IBVP problem for the compressible Navier-Stokes equations (2.1)-(2.2) with initial data (2.3) and boundary condition (2.6) or (2.7), but also that this short time entropy weak solution satisfies all the properties (2.23) $-(2.32)$. Now, choose time $T_{*}=T_{*}^{\prime}-\delta$ with $\delta>0$ a constant small enough. One can verify that at time $t=T_{*}$ the density and momentum also satisfies the assumptions (2.4), and particularly

$$
\left\{\begin{array}{l}
\tilde{\rho}\left(x, T_{*}\right) \geq 0 \text { on } \Omega, \quad \tilde{\rho} \tilde{u}\left(., T_{*}\right)=0, \quad \text { on }\left\{x \in \Omega \mid \rho_{0}(x)=0\right\} \\
\tilde{\rho}\left(., T_{*}\right) \in L^{1}(\Omega), \quad\left(\tilde{\rho}^{\alpha-1 / 2}\left(., T_{*}\right)\right)_{x} \in L^{2}(\Omega), \\
\frac{\left|\tilde{m}\left(., T_{*}\right)\right|^{2}}{\tilde{\rho}\left(x, T_{*}\right)}+\frac{\left|\tilde{m}\left(., T_{*}\right)\right|^{2+\nu}}{\tilde{\rho}^{1+\nu}\left(x, T_{*}\right)} \in L^{1}(\Omega) .
\end{array}\right.
$$

Thus, it follows from the Theorem 2.1 that there is a global entropy weak solution $(\hat{\rho}, \hat{u})$ for time $t \geq T_{*}$ in the sense of Definition 2.5 to the IBVP problem for the compressible Navier-Stokes equations (2.1)-(2.2) with initial data

$$
(\rho, \rho u)\left(x, T_{*}\right)=(\tilde{\rho}, \tilde{\rho} \tilde{u})\left(x, T_{*}\right), \quad x \in \Omega
$$

and boundary condition (2.6) or (2.7). Define $(\rho, u)$ as

$$
(\rho, u)= \begin{cases}(\tilde{\rho}, \tilde{u}), & \text { for } \quad(x, t) \in \Omega \times\left[0, T_{*}\right], \\ (\hat{\rho}, \hat{u}), & \text { for } \quad(x, t) \in \Omega \times\left[0, T_{*}\right] .\end{cases}
$$

It is easy to verify that $(\rho, u)$ is a global entropy weak solution in the sense of Definition 2.5 to the compressible Navier-Stokes equations (2.1)-(2.2) with initial data (2.3) and boundary condition (2.6) or (2.7) under the assumptions of Theorem 2.2. This global entropy weak solution is actually unique and regular for short time $t \in\left[0, T_{*}\right]$ and satisfies all the properties (2.23) $-(2.32)$. The proof of Theorem 2.2 is completed. 


\subsection{Compressible Navier-Stokes in Lagrangian coordinates}

In order to prove the Proposition 4.1, we present an equivalent proposition for the compressible Navier-Stokes equations in the Lagrangian coordinates $(y, t)$, instead of the Eulerian coordinates $(x, t)$, through the coordinate transformation

$$
y=\int_{0}^{x} \rho(z, t) d z, \quad x \in(0,1), \quad t \geq 0
$$

for the Dirichlet boundary condition, or

$$
y=\int_{X_{0}(t)}^{X_{0}(t)+x} \rho(z, t) d z, \quad x \in(0,1), \quad t \geq 0
$$

for the periodic boundary condition where $x=X_{0}(t)$ is a particle path. In addition, both the case of initial one point vacuum state and the case of a piece of initial continuous vacuum states will be studied with some additional initial regularities.

We first describe the equivalent proposition for the IBVP problem for the compressible Navier-Stokes equations in the Lagrangian coordinates in the case of initial one point vacuum state. Thus, consider the compressible Navier-Stokes equations

$$
\begin{aligned}
\rho_{t}+\rho^{2} u_{y} & =0, \\
u_{t}+p(\rho)_{y}-\left(\rho \mu(\rho) u_{y}\right)_{y} & =0, \quad y \in \Omega, t>0
\end{aligned}
$$

with initial data

$$
(\rho, u)(y, 0)=\left(\rho_{0}(y), u_{0}(y)\right), \quad y \in \Omega,
$$

and the Dirichlet boundary condition

$$
u(0, t)=u(1, t)=0, \quad t \geq 0
$$

or the periodic boundary condition

$$
(\rho, u) \text { is periodic w.r.t. } x \text { of period one. }
$$

For the case of Dirichlet boundary, the initial data $\left(\rho_{0}, u_{0}\right)$ is assumed to be consistent with the boundary values. Moreover, we assume that there is one point vacuum state at $y=y_{0}$ for some fixed point $y_{0} \in(0,1)$ and that the initial data is of additional regularity

$$
\left\{\begin{array}{l}
A_{0}\left|y-y_{0}\right|^{\beta} \leq \rho_{0}(y) \leq A_{1}\left|y-y_{0}\right|^{\beta}, \quad y \in \Omega, \\
u_{0} \in C^{1}(\bar{\Omega}), \quad\left(\rho_{0}^{\gamma}\right)_{y},\left(\rho_{0}^{1+\alpha} u_{0 y}\right)_{y} \in L^{2}(\Omega) \cap L^{2 n}(\Omega)
\end{array}\right.
$$

with an integer $n \geq 2$, where $A_{0}, A_{1}$ are positive constant, and the constant $\beta \in$ $\left(\beta_{-}, \beta_{+}\right)$with $\beta_{ \pm}$determined by Remark 4.3. Then the following result holds in the case of initial point vacuum state. 
Proposition 4.2 (Point vacuum state for short time) Assume that (2.22) and (4.12) hold. Then, there is a time $T_{*}^{\prime}>0$ so that the unique regular weak solution $(\rho, u)$ with point vacuum of the IBVP (4.7) - (4.9) with boundary condition (4.10) or (4.11) exists on the domain $\Omega \times\left[0, T_{*}^{\prime}\right]$ and satisfies

$$
\begin{gathered}
\rho \in C^{0}\left(\bar{\Omega} \times\left[0, T_{*}^{\prime}\right]\right) \cap C^{1}\left(\left[0, T_{*}^{\prime}\right] ; L^{2}(\Omega)\right), \\
u \in C^{0}\left(\bar{\Omega} \times\left[0, T_{*}^{\prime}\right]\right) \cap C^{1}\left(\left[0, T_{*}^{\prime}\right] ; L^{2}(\Omega)\right), \\
\rho^{1+\alpha} u_{y} \in L^{\infty}\left(\Omega \times\left[0, T_{*}^{\prime}\right]\right) \cap C^{1 / 2}\left(\left[0, T_{*}^{\prime}\right] ; L^{2}(\Omega)\right), \\
\left\|\left(\rho^{\alpha}\right)_{y}\right\|_{L^{\infty}\left(\left[0, T_{*}^{\prime}\right] ; L^{2}(\Omega)\right)}+\left\|\rho u_{y}\right\|_{L^{\infty}\left(\Omega \times\left[0, T_{*}^{\prime}\right]\right)} \leq C\left(T_{*}\right) .
\end{gathered}
$$

In addition, the initial point vacuum state is maintained for the short time

$$
\begin{gathered}
\rho\left(y_{0}, t\right)=0, \quad t \in\left[0, T_{*}^{\prime}\right] \\
a_{-}\left|y-y_{0}\right|^{\beta} \leq \rho(y, t) \leq a_{+}\left|y-y_{0}\right|^{\beta},(y, t) \in[0,1] \times\left[0, T_{*}\right] .
\end{gathered}
$$

Here, $a_{ \pm}$are positive constants independent of $T_{*}^{\prime}$.

Proof: The proof of the Proposition 4.1 will be given in subsection 4.3,

Remark 4.3 The choice of $\beta_{ \pm}>0$ is such that

$$
\beta_{-}=\max \left\{\frac{1}{2 \alpha}, \frac{1}{\gamma}\left(1-\frac{1}{2 n}\right)\right\}, \quad \beta_{+}=\min \left\{1, \frac{1}{\alpha}\left(1-\frac{1}{2 n}\right), \frac{1}{1+3 \alpha}\left(4-\frac{1}{n}\right)\right\}
$$

for integer $n \geq 2$. It should be emphasized here that all the assumptions required here are satisfied for the shallow water equations (1.2), i.e., $\gamma=2, \alpha=1$.

Nest, we describe the equivalent proposition for the IBVP problem for the compressible Navier-Stokes equations in the Lagrangian coordinates for the case of initial continuous vacuum of one piece. In this case, we consider the IBVP problem for the compressible Navier-Stokes equation (4.7)-(4.9) with one of following boundary conditions:

(1). mixed boundary condition

$$
\left\{\begin{array}{l}
u(0, t)=\rho(1, t)=0, \quad t \geq 0 \\
A_{0}(1-y)^{\beta} \leq \rho_{0}(y) \leq A_{1}(1-y)^{\beta}, y \in \Omega
\end{array}\right.
$$

or

(2). mixed free boundary condition

$$
\left\{\begin{array}{l}
\rho(0, t)=u(1, t)=0, \quad t \geq 0 \\
B_{0} y^{\beta} \leq \rho_{0}(y) \leq B_{1} y^{\beta}, \quad y \in \Omega
\end{array}\right.
$$

or 
(3). free boundary condition

$$
\left\{\begin{array}{l}
\rho(0, t)=\rho(1, t)=0, t \geq 0 \\
A_{0}^{\prime}(y(1-y))^{\beta} \leq \rho_{0}(y) \leq A_{1}^{\prime}(y(1-y))^{\beta}, y \in \Omega
\end{array}\right.
$$

where, $\beta \in\left(\beta_{-}, \beta_{+}\right)$, and $A_{0}, A_{1}, B_{0}, B_{1}$, and $A_{0}^{\prime}, A_{1}^{\prime}$ are some given positive constants. The initial data is also assumed to be regular

$$
u_{0} \in C^{1}(\bar{\Omega}), \quad\left(\rho_{0}^{\gamma}\right)_{y}, \quad\left(\rho_{0}^{1+\alpha} u_{0 y}\right)_{y} \in L^{2}(\Omega) \cap L^{2 n}(\Omega)
$$

with an integer $n \geq 2$. Then the following results for free boundary value problems for the compressible Navier-Stokes equations hold.

Proposition 4.4 (Continuous vacuum state for short time) Assume that (2.22) and (4.23) hold. Then, there is a time $T_{*}^{\prime}>0$ so that the unique regular weak solution $(\rho, u)$ of the IBVP problem for the compressible Navier-Stokes equations (4.7) - (4.8) with initial data (4.9) and free boundary condition (4.20), or (4.21), or (4.22) exists on the domain $\Omega \times\left[0, T_{*}^{\prime}\right]$ and satisfies

$$
\begin{gathered}
\rho \in C^{0}\left(\bar{\Omega} \times\left[0, T_{*}^{\prime}\right]\right) \cap C^{1}\left(\left[0, T_{*}^{\prime}\right] ; L^{2}(\Omega)\right), \\
u \in C^{0}\left(\bar{\Omega} \times\left[0, T_{*}^{\prime}\right]\right) \cap C^{1}\left(\left[0, T_{*}^{\prime}\right] ; L^{2}(\Omega)\right), \\
\rho^{1+\alpha} u_{y} \in L^{\infty}\left(\Omega \times\left[0, T_{*}^{\prime}\right]\right) \cap C^{1 / 2}\left(\left[0, T_{*}^{\prime}\right] ; L^{2}(\Omega)\right),
\end{gathered}
$$

and

$$
\left\|\left(\rho^{\alpha}\right)_{y}\right\|_{L^{\infty}\left(\left[0, T_{*}^{\prime}\right] ; L^{2}(\Omega)\right)}+\left\|\rho u_{y}\right\|_{L^{\infty}\left(\Omega \times\left[0, T_{*}^{\prime}\right]\right)} \leq C\left(T_{*}^{\prime}\right) .
$$

In addition, the initial vacuum state is also maintained for short time, namely, it holds that

$$
a_{-}(1-y)^{\beta} \leq \rho(y, t) \leq a_{+}(1-y)^{\beta}, \quad(y, t) \in(0,1) \times\left[0, T_{*}^{\prime}\right]
$$

corresponding to the mixed free boundary conditions 4.20, or

$$
b_{-} y^{\beta} \leq \rho(y, t) \leq b_{+} y^{\beta}, \quad(y, t) \in(0,1) \times\left[0, T_{*}^{\prime}\right]
$$

corresponding to the mixed free boundary conditions (4.21), or

$$
c_{-}(y(1-y))^{\beta} \leq \rho(y, t) \leq c_{+}(y(1-y))^{\beta}, \quad(y, t) \in(0,1) \times\left[0, T_{*}^{\prime}\right]
$$

corresponding to the free boundary (4.22). Here, $a_{ \pm}, b_{ \pm}$and $c_{ \pm}$are positive constants independent of $T_{*}^{\prime}$.

Proof: The short time existence, uniqueness and regularity of weak solutions of the free boundary problems for the compressible Navier-Stokes equations are wellinvestigated by many authors (see $[23,32,33,56,57]$ ). Proposition 4.4 can be proved in a similar way as $[23,32,33,56]$, so we omit the details. 
Remark 4.5 Based on Proposition 4.4 for the compressible Navier-Stokes equations (4.7) - (4.9) with either mixed free boundary condition (4.20) and (4.21) or free boundary (4.22) and the coordinates transformation from the Lagrangian coordinates to the Eulerian coordinates, one can study easily the IBVP problem for compressible Navier-Stokes equations (2.1) -(2.2) in the Eulerian coordinates with either the Dirichlet boundary condition (2.6) or the periodic boundary condition (2.7) in the case of initial continuous vacuum state of one piece (2.20).

In fact, for the compressible Navier-Stokes equations (2.1)-(2.2) with Dirichlet boundary condition (2.6) in the Eulerian coordinates, the short time existence of unique solution subject to the case of a piece of initial continuous vacuum state (2.20) -(2.21) in initial data can be constructed, in the Lagrangian coordinates, by combining two mixed free boundary value problems (4.20) and (4.21) together with one continuous vacuum state in-between as follows. Denote two particle pathes $x=X_{i}(t)$ (assumed to be definable for short time) starting from the initial vacuum boundary $x=x_{0}$ and $x=x_{1}$ respectively as

$$
\dot{X}_{i}(t)=u\left(X_{i}(t), t\right), \quad X_{i}(0)=x_{i}, i=0,1,
$$

along which the vacuum boundary moves in the Eulerian coordinates so that

$$
\begin{cases}(\rho, \rho u)(x, t)=0, & x \in\left[X_{0}(t), X_{1}(t)\right], t \geq 0, \\ \rho u(x, t)>0, & x \in\left[0, X_{0}(t)\right) \cup\left(X_{1}(t), 1\right], t \geq 0 .\end{cases}
$$

We first choose the coordinate transformation from the Eulerian coordinates to the Lagrangian coordinates as

$$
\left\{\begin{array}{l}
y=\int_{0}^{x} \rho(z, t) d z, \quad x \in\left[0, X_{0}(t)\right] \\
y_{0}=\int_{0}^{X_{0}(t)} \rho(z, t) d z=\int_{0}^{x_{0}} \rho_{0}(z) d z<1, \quad \text { conservation of mass }
\end{array}\right.
$$

which gives $y \in\left[0, y_{0}\right]$ and the mixed free boundary conditions (4.20). The application of the Proposition 4.4 with $\Omega$ replaced by $\left(0, y_{0}\right)$ implies, via the inverse coordinate transformation $x=\int_{0}^{y} \rho^{-1}(z, t) d z, y \in\left[0, y_{0}\right]$, the existence of unique solution $\left(\rho_{l}, u_{l}\right)$ of the compressible Navier-Stokes equations (2.1)-(2.2) on $\left[0, X_{0}(t)\right] \times\left[0, T_{*}\right]$ in the Eulerian coordinates with the initial data

$$
\left(\rho_{l}, u_{l}\right)(x, 0)=\left(\rho_{0}, u_{0}\right)(x), \quad x \in\left(0, x_{0}\right), \quad \rho_{0}\left(x_{0}\right)=0,
$$

and mixed free boundary conditions

$$
u(0, t)=0, \quad \rho\left(X_{0}(t), t\right)=0, \quad t \in\left[0, T_{*}\right]
$$

Next, we choose the coordinate transformation from the Eulerian coordinates to the Lagrangian coordinates as

$$
\left\{\begin{array}{l}
y=1-\int_{x}^{1} \rho(z, t) d z, \quad x \in\left[X_{1}(t), 1\right] \\
y_{1}=\int_{X_{1}(t)}^{1} \rho(z, t) d z=\int_{x_{1}}^{1} \rho_{0}(z) d z<1 \quad \text { conservation of mass }
\end{array}\right.
$$


which gives $y \in\left[1-y_{1}, 1\right]$ and the mixed free boundary conditions [4.21]. Then Proposition 4.4 implies, via the inverse coordinate transformation $x=1-\int_{y}^{1} \rho^{-1}(z, t) d z$, $y \in\left[1-y_{1}, 1\right]$, the existence of unique $\left(\rho_{r}, u_{r}\right)$ of the compressible Navier-Stokes equations (2.1) -(2.2) on $\left[X_{1}(t), 1\right] \times\left[0, T_{*}\right]$ in the Eulerian coordinates with initial data

$$
\left(\rho_{r}, u_{r}\right)(x, 0)=\left(\rho_{0}, u_{0}\right)(x), \quad x \in\left(x_{1}, 1\right), \quad \rho_{0}\left(x_{1}\right)=0,
$$

and mixed free boundary conditions

$$
u(1, t)=0, \quad \rho\left(X_{1}(t), t\right)=0, \quad t \in\left[0, T_{*}\right] .
$$

Consequently, we can construct the short time unique solution $(\rho, u)$ to the IBVP problem for the compressible Navier-Stokes equations (2.1) -(2.3) with the Dirichlet boundary condition (2.6) and a piece of continuous vacuum state (2.20) in the initial data as

$$
(\rho, \rho u)=\left\{\begin{array}{lll}
\left(\rho_{l}, \rho_{l} u_{l}\right), & \text { on } & {\left[0, X_{0}(t)\right] \times\left[0, T_{*}\right],} \\
(0,0), & \text { on } & \left(X_{0}(t), X_{1}(t)\right) \times\left[0, T_{*}\right], \\
\left(\rho_{r}, \rho_{r} u_{r}\right), & \text { on } & {\left[X_{1}(t), 1\right] \times\left[0, T_{*}\right]}
\end{array}\right.
$$

Similarly, we can obtain the short time existence of unique solution to the IBVP problem for the compressible Navier-Stokes equations (2.1) -(2.2) with periodic boundary condition (2.7) which can be viewed as a free boundary problem after the choice of the spatial reference point. The details will be omitted.

\subsection{Proof of Propositions 4.14 .2}

We first prove the Proposition 4.2 in this subsection with the help of the a-priori estimates for (regularized) solutions and the construction of approximate solutions by a finite difference scheme, due to the modification of the ideas used in $[23,33,43,56]$. Without the loss of generality, we only prove Proposition 4.2 in the case of the Dirichlet boundary conditions with one point vacuum state in the initial data.

First, we can easily derive some identities for (regularized) solutions as in $[23,56]$.

Lemma 4.6 Let $T>0$ and assume that the solution $(\rho, u)$ of the IBVP problem (4.7)(4.10) exists for $t \in[0, T]$ with $\rho\left(y_{0}, t\right)=0$. Then, under the assumptions of Proposition 4.2. it holds that

$$
\begin{array}{r}
\rho^{1+\alpha} u_{y}(y, t)=\int_{y_{0}}^{y} u_{t}(z, t) d z+\rho^{\gamma}(y, t)=-\int_{y}^{y_{0}} u_{t}(z, t) d z+\rho^{\gamma}(y, t), \\
\rho^{\alpha}(y, t)+\alpha \int_{0}^{t} \rho^{\gamma}(y, s) d s=\rho_{0}^{\alpha}(y)+\alpha \int_{0}^{t} \int_{y}^{y_{0}} u_{t}(z, s) d z d s \\
=\rho_{0}^{\alpha}(y)-\alpha \int_{0}^{t} \int_{y_{0}}^{y} u_{t}(z, s) d z d s .
\end{array}
$$

for all $y \in \Omega$ with $y \neq y_{0}$. 
We also have the following useful a-priori estimates, whose proofs are similar to those for (3.11) and (3.19) and thus will be omitted.

Lemma 4.7 Let $T>0$ and assume that the solution $(\rho, u)$ of the IBVP problem (4.7)(4.10) exists for $t \in[0, T]$. Then, under the assumptions of Proposition 4.2, it holds that

$$
\begin{gathered}
\|u(t)\|_{L^{2}(\Omega)}^{2}+\|\rho(t)\|_{L^{\gamma-1}(\Omega)}^{\gamma-1}+\int_{0}^{t} \int\left|\left(\rho^{(\gamma+\alpha) / 2}\right)_{y}\right|^{2} d x d s \leq C_{0}, \quad t \in[0, T] \\
\|\rho(t)\|_{L^{\infty}(\Omega)}+\left\|\left(\rho^{\alpha}(t)\right)_{y}\right\|_{L^{2}(\Omega)} \leq C_{0}, \quad t \in[0, T]
\end{gathered}
$$

with $C_{0}>0$ a constant.

Lemma 4.8 Let $T>0$ and assume that the solution $(\rho, u)$ of the IBVP problem (4.7)(4.10) exists for $t \in[0, T]$ with $\rho\left(y_{0}, t\right)=0$. Then, under the assumptions of Proposition 4.2. there is a time $T_{*}^{\prime} \in(0, T]$ (depending on initial data) so that it holds that

$$
\int u_{t}^{2 j}(y, t) d y+\int_{0}^{t} \int u_{t}^{2 j-2} \rho^{1+\alpha} u_{y t}^{2} d x d s \leq C_{1}
$$

uniformly for $t \in\left[0, T_{*}^{\prime}\right]$ with $j=1, n$ and $C_{1}>0$ a positive constant, and that $\rho u_{y}(t) \in C^{0}(\bar{\Omega})$ is uniformly bounded for any $t \in\left[0, T_{*}^{\prime}\right]$

$$
\left\|\rho u_{y}\right\|_{L^{\infty}\left(\Omega \times\left[0, T_{*}^{\prime}\right]\right)} \leq C_{2}
$$

with $C_{2}>0$ a constant. Moreover, the solution $(\rho, u)$ is continuous

$$
(\rho, u) \in C^{0}\left(\bar{\Omega} \times\left[0, T_{*}^{\prime}\right]\right)
$$

and the initial one point vacuum state is maintained

$$
a_{-}\left|y-y_{0}\right|^{\beta} \leq \rho(y, t) \leq a_{+}\left|y-y_{0}\right|^{\beta}, \quad(y, t) \in \Omega \times\left[0, T_{*}^{\prime}\right]
$$

with $a_{+}>a_{-}>0$ two constants independent of time $T_{*}$.

Proof: Let us first assume that the weak solution is regular enough so that we can differentiate it through the equations and the interface as in [56], and the density is of the form

$$
a_{*}\left|y-y_{0}\right|^{\beta} \leq \rho(y, t) \leq a^{*}\left|y-y_{0}\right|^{\beta}, \quad(y, t) \in \Omega \times[0, T]
$$

with $a_{*}, a^{*}$ two positive constants to be determined later. It will be assumed further that the following a-priori estimate holds

$$
\left\|\rho u_{y}\right\|_{L^{\infty}(\Omega \times[0, T])} \leq M_{0}
$$


for some positive $M_{0}$ to be determined later. We will prove (4.30) only for $j=n$ below since the other case can be treated similarly. Taking inner product between (4.8) $t$ and $2 n\left(u_{t}\right)^{2 n-1}$ over $\Omega$ leads to

$$
\frac{d}{d t} \int\left|u_{t}\right|^{2 n} d y+2 n \int\left(\rho^{\gamma}\right)_{y t}\left(u_{t}\right)^{2 n-1} d y-2 n \int\left(\rho^{1+\alpha} u_{y}\right)_{y t}\left(u_{t}\right)^{2 n-1} d y=0
$$

from which, one deduces after integration by parts and using Eq. (4.7) that

$$
\begin{aligned}
& \frac{d}{d t} \int\left|u_{t}\right|^{2 n} d y+2 n(2 n-1) \int \rho^{1+\alpha} u_{y t}^{2}\left(u_{t}\right)^{2 n-2} d y \\
= & 2 n(2 n-1) \gamma \int \rho^{\gamma-1} \rho_{t} u_{y t}\left(u_{t}\right)^{2 n-1} d y \\
& +2 n(2 n-1)(1+\alpha) \int \rho^{\alpha} \rho_{t} u_{y} u_{y t}\left(u_{t}\right)^{2 n-2} d y \\
\leq & n(2 n-1) \int \rho^{1+\alpha} u_{y t}^{2}\left(u_{t}\right)^{2 n-2} d y+C C_{0}^{2 \gamma-1-\alpha} M_{0}^{2}\left(1+\left\|u_{t}(t)\right\|_{L^{2 n-2}}^{2 n+2}\right) \\
& +C \int \rho^{3+\alpha} u_{y}^{4}\left(u_{t}\right)^{2 n-2} d y
\end{aligned}
$$

where one has used (2.22), (4.29), the a-priori assumption (4.35), and Hölder's inequality. The last term on the right hand side of (4.36) can be estimated as follows. For the case $\alpha \geq 1$, it follows from (4.29) and (4.35) that

$$
\begin{aligned}
\int \rho^{3+\alpha} u_{y}^{4}\left(u_{t}\right)^{2 n-2} d y & \leq\left\|\rho^{3+\alpha} u_{y}^{4}\right\|_{L^{\infty}}\left\|u_{t}(t)\right\|_{L^{2 n-2}}^{2 n-2} \leq C_{0}^{\alpha-1} M_{0}^{4}\left\|u_{t}(t)\right\|_{L^{2 n-2}}^{2 n-2} \\
& \leq C M_{0}^{4}\left(1+\left\|u_{t}(t)\right\|_{L^{2 n}}^{2 n+2}\right) .
\end{aligned}
$$

For the case $\alpha \in\left(\frac{1}{2}, 1\right)$, since it holds by (4.27) that

$$
\left|\rho^{1+\alpha} u_{y}\right| \leq\left|\int_{y_{0}}^{y} u_{t}(z, t) d z\right|+\rho^{\gamma} \leq\left\|u_{t}\right\|_{L^{2 n}} \cdot\left|y-y_{0}\right|^{(2 n-1) / 2 n}+\rho^{\gamma}
$$

which together (4.34) implies

$$
\begin{aligned}
\left|\rho^{(3+\alpha) n} u_{y}^{4 n}\right| & =\left|\rho^{-(1+3 \alpha) n}\left(\rho^{1+\alpha} u_{y}\right)^{4 n}\right|=\rho^{-(1+3 \alpha) n}\left[\int_{y_{0}}^{y} u_{t}(z, t) d z+\rho^{\gamma}\right]^{4 n} \\
& \leq C \rho^{-(1+3 \alpha) n}\left(\left\|u_{t}\right\|_{L^{2 n}}^{4 n} \cdot\left|y-y_{0}\right|^{2(2 n-1)}+\rho^{4 n \gamma}\right) \\
& \leq C\left\|u_{t}\right\|_{L^{2 n}}^{4 n} \cdot\left|y-y_{0}\right|^{-(1+3 \alpha) n \beta+2(2 n-1)}+\rho^{3 n(\gamma-\alpha)+n(\gamma-1)}
\end{aligned}
$$

the last term in (4.36) is estimated by

$$
\left|\int \rho^{3+\alpha} u_{y}^{4}\left(u_{t}\right)^{2 n-2} d y\right| \leq\left\|u_{t}\right\|_{L^{2 n}}^{2 n-2} \cdot\left(\int \rho^{(3+\alpha) n} u_{y}^{4 n} d y\right)^{1 / n}
$$




$$
\begin{aligned}
& \leq C\left\|u_{t}\right\|_{L^{2 n}}^{2 n-2} \cdot\left(\left\|u_{t}\right\|_{L^{2 n}}^{4 n} \int\left|y-y_{0}\right|^{-(1+3 \alpha) n \beta+2(2 n-1)} d y+\int \rho^{3 n(\gamma-\alpha)+n(\gamma-1)} d y\right)^{1 / n} \\
& \leq C\left(\left\|u_{t}\right\|_{L^{2 n}}^{2 n+2}+1\right) .
\end{aligned}
$$

where one has used the fact $\beta<\frac{4 n-1}{n(1+3 \alpha)}$ due to (4.19). Substituting (4.37) and (4.40) into (4.36) shows

$$
\begin{aligned}
& \frac{d}{d t} \int\left|u_{t}\right|^{2 n} d y+n(2 n-1) \int \rho^{1+\alpha} u_{y t}^{2}\left(u_{t}\right)^{2 n-2} d y \\
\leq & C M_{0}^{2}\left(1+M_{0}^{2}\right)\left(1+\left\|u_{t}(t)\right\|_{L^{2 n-2}}^{2 n+2}\right) \leq C\left(1+M_{0}^{4}\right)\left(\left\|u_{t}(t)\right\|_{L^{2 n}}^{2 n+2}+1\right) .
\end{aligned}
$$

Set

$$
T_{a}=\min \left\{\frac{2^{n}-1}{n 2^{n} C\left(1+M_{0}^{4}\right) C_{1}}, \quad \frac{\left\|u_{t}(0)\right\|_{L^{2 n}}^{2 n}}{C\left(1+M_{0}^{4}\right)}, \quad T\right\} .
$$

One can apply the Grönwall's Lemma to obtain (4.30) for $t \in\left[0, T_{a}\right]$ with $C_{1}$ given by

$$
C_{1}=: 2\left\|u_{t}(0)\right\|_{L^{2 n}}^{2 n} \geq\left\|u_{t}(0)\right\|_{L^{2 n}}^{2 n}+C T_{a}\left(1+M_{0}^{4}\right) .
$$

To prove (4.31) and ensure the a-priori assumption (4.35), we use the equality (4.27) to get that near $y=y_{0}$, it holds that

$$
\rho u_{y}(y, t)=\rho^{-\alpha}(y, t) \int_{y_{0}}^{y} u_{t}(z, t) d z+\rho^{\gamma-\alpha}(y, t), \quad y \neq y_{0}
$$

which, together with (2.22), (4.29), and the fact $\beta \leq \frac{1}{\alpha}\left(1-\frac{1}{2 n}\right)$ due to (4.19), implies

$$
\begin{aligned}
\left\|\rho u_{y}\right\|_{L_{\Omega \times[0, t]}^{\infty}} & \leq C_{0}^{\gamma-\alpha}+\left|\rho^{-\alpha}(y, t) \int_{y_{0}}^{y} u_{t}(z, t) d z\right|_{L_{\Omega \times[0, t]}^{\infty}} \\
& \leq C_{0}^{\gamma-\alpha}+a_{*}^{-\alpha}\left|y-y_{0}\right|^{1-1 / 2 n-\alpha \beta}\left\|u_{t}(t)\right\|_{L^{2 n}} \\
& \leq C_{0}^{\gamma-\alpha}+a_{*}^{-\alpha} C_{1}^{1 / 2 n} \leq C_{0}^{\gamma-\alpha}+2 a_{*}^{-\alpha}\left\|u_{t}(0)\right\|_{L^{2 n}} \\
& =: C_{2} \leq M_{0}
\end{aligned}
$$

so long as the constant $M_{0}$ is chosen as

$$
M_{0}=1+C_{0}^{\gamma-\alpha}+2 a_{*}^{-\alpha}\left\|u_{t}(0)\right\|_{L^{2 n}} .
$$

Next, we verify the a-priori assumption (4.34). Set

$$
T_{b}=\min \left\{\frac{A_{0}^{\alpha}}{3 \alpha C_{1}^{1 / 2 n}}, \quad T_{a}\right\} .
$$

It follows from the equation (4.28) for $y \neq y_{0}$ and $t \in\left[0, T_{b}\right]$ that

$$
\begin{aligned}
& \rho^{\alpha}(y, t)+\alpha \int_{0}^{t} \rho^{\gamma}(y, s) d s=\rho_{0}^{\alpha}(y)+\alpha \int_{0}^{t} \int_{y_{0}}^{y} u_{t}(z, s) d z d s \\
\geq & \frac{2}{3} A_{0}^{\alpha}\left|y-y_{0}\right|^{\alpha \beta}+\left|y-y_{0}\right|^{\alpha \beta}\left(\frac{1}{3} A_{0}^{\alpha}-C_{1}^{1 / 2 n} \alpha T_{b}\left|y-y_{0}\right|^{1-1 / 2 n-\alpha \beta}\right) \\
\geq & \frac{2}{3} A_{0}^{\alpha}\left|y-y_{0}\right|^{\alpha \beta}
\end{aligned}
$$


where we have used the facts $\left|y-y_{0}\right| \leq 1$ and $\beta \leq \frac{1}{\alpha}\left(1-\frac{1}{2 n}\right)$ due to (4.19). On the other hand, it follows from (4.28) that

$$
\begin{aligned}
& \rho^{\alpha}(y, t)+\alpha \int_{0}^{t} \rho^{\gamma}(y, s) d s=\rho_{0}^{\alpha}(y)+\alpha \int_{0}^{t} \int_{y_{0}}^{y} u_{t}(z, s) d z d s \\
\leq & A_{1}^{\alpha}\left|y-y_{0}\right|^{\alpha \beta}+\alpha t\left|y-y_{0}\right|^{1-1 / 2 n}\left\|u_{t}(t)\right\|_{L^{2 n}} \\
\leq & \left(A_{1}^{\alpha}+C_{1}^{1 / 2 n} \alpha t\right)\left|y-y_{0}\right|^{\alpha \beta} .
\end{aligned}
$$

Define $T_{c} \in\left(0, T_{b}\right]$ by

$$
T_{c}=\min \left\{\frac{A_{0}^{\alpha}}{3 \alpha C\left(A_{1}^{\alpha}+C_{1}^{1 / 2 n} \alpha T_{b}\right)^{\gamma / \alpha}}, \quad T_{a}, T_{b}\right\} .
$$

Set

$$
Z(t)=\int_{0}^{t} \rho^{\gamma}(y, s) d s
$$

It follows from (4.48) that

$$
\left(Z^{\prime}(t)\right)^{\alpha / \gamma} \leq\left(A_{1}^{\alpha}+C_{1}^{1 / 2 n} \alpha t\right)\left|y-y_{0}\right|^{\alpha \beta}
$$

which implies for $t \in\left[0, T_{c}\right]$ that

$$
\begin{aligned}
\int_{0}^{t} \rho^{\gamma}(y, s) d s & \leq T_{c}\left(A_{1}^{\alpha}+C_{1}^{1 / 2 n} \alpha T_{c}\right)^{\gamma / \alpha}\left|y-y_{0}\right|^{(\gamma-\alpha) \beta}\left|y-y_{0}\right|^{\alpha \beta} \\
& \leq C T_{c}\left(A_{1}^{\alpha}+C_{1}^{1 / 2 n} \alpha T_{b}\right)^{\gamma / \alpha}\left|y-y_{0}\right|^{\alpha \beta} .
\end{aligned}
$$

As a consequence of (4.47), (4.50), and (4.49), one gets

$$
\rho^{\alpha}(y, t) \geq \frac{2}{3} A_{0}^{\alpha}\left|y-y_{0}\right|^{\alpha \beta}-\alpha \int_{0}^{t} \rho^{\gamma}(y, s) d s \geq \frac{1}{3} A_{0}^{\alpha}\left|y-y_{0}\right|^{\alpha \beta} .
$$

From (4.48) and (4.51), we can verify the a-priori assumption (4.34) and justify the property (4.33) by simply choosing

$$
a_{*}=a_{-}=\left(A_{0}^{\alpha} / 3\right)^{1 / \alpha}, \quad a^{*}=a_{+}=\left(A_{1}^{\alpha}+C_{1}^{1 / 2 n} \alpha T_{c}\right)^{1 / \alpha}
$$

for any $t \in\left[0, T_{*}^{\prime}\right]$ with time $T_{*}^{\prime}=T_{c}$ determined by (4.49). One then derives from Eq. (4.7), (4.29), and (4.31) that

$$
\rho^{\alpha} \in L^{\infty}\left(0, T_{*}^{\prime}, H^{1}(\Omega)\right), \quad\left(\rho^{\alpha}\right)_{t} \in L^{\infty}\left(0, T_{*}^{\prime}, L^{2}(\Omega)\right) .
$$

while (4.31), (4.33), boundary condition (4.10), and (4.30) for $j=1$ imply that

$$
u \in L^{\infty}\left(0, T_{*}^{\prime}, W_{0}^{1, p}(\Omega)\right), \quad u_{t} \in L^{\infty}\left(0, T_{*}^{\prime}, L^{2}(\Omega)\right)
$$


for any $p \in\left(1, \beta^{-1}\right)$, where one has used the fact $\beta^{-1}>\beta_{+}^{-1} \geq 1$ so that

$$
\sup _{t \in\left[0, T_{*}\right]}\left\|u_{y}\right\|_{L^{p}(\Omega)}=\sup _{t \in\left[0, T_{*}\right]}\left\|\rho^{-p}\right\|_{L^{1}(\Omega)} \cdot\left\|\rho u_{y}\right\|_{L^{\infty}\left(\Omega \times\left[0, T_{*}^{\prime}\right]\right)}^{p} \leq C .
$$

(4.53) - 4.55) imply the continuity (4.32) of the solution $(\rho, u)$, and the continuity of $\rho u_{y}$ follows from the equation (4.27) and that of $(\rho, u)$. The proof of the lemma is completed.

Using Lemmas4.64.8 and a direct computation, we can obtain the following result:

Lemma 4.9 Let $T_{*}^{\prime}>0$ be given in Lemma 4.8 and $(\rho, u)$ be the solution of the IBVP problem (4.7)-(4.10). Then, under the assumptions of Proposition 4.2, $\rho^{1+\alpha} u_{y}(t) \in$ $C^{0}(\bar{\Omega})$ is uniformly bounded for any $t \in\left[0, T_{*}^{\prime}\right]$

$$
\lim _{y \rightarrow y_{0}} \rho^{1+\alpha} u_{y}(y, t)=0, \quad\left\|\rho^{1+\alpha} u_{y}\right\|_{L^{\infty}\left(0, T_{*}^{\prime} ; C^{0}(\bar{\Omega})\right)} \leq C\left(T_{*}^{\prime}\right),
$$

and $(\rho, u)$ satisfies for $0 \leq s<t \leq T_{*}^{\prime}$ that

$$
\begin{gathered}
\|\rho(t)-\rho(s)\|_{L^{2}(\Omega)}+\|u(t)-u(s)\|_{L^{2}(\Omega)} \leq C\left(T_{*}^{\prime}\right)|t-s|, \\
\left\|\rho^{1+\alpha} u_{y}(t)-\rho^{1+\alpha} u_{y}(s)\right\|_{L^{2}(\Omega)} \leq C\left(T_{*}^{\prime}\right)|t-s|^{1 / 2}
\end{gathered}
$$

Proof: The facts that for any $t \in\left[0, T_{*}\right]$, it holds that $\rho^{1+\alpha} u_{y}(t) \in C^{0}(\bar{\Omega})$ and $\lim _{y \rightarrow y_{0}} \rho^{1+\alpha} u_{y}(y, t)=0$ are due to the continuity of right hand side terms of (4.44) and (4.34). By (4.31) and (4.33), one can check easily that

$$
\left\|\rho^{1+\alpha} u_{y}\right\|_{L^{\infty}\left(\Omega \times\left[0, T_{*}^{\prime}\right]\right)} \leq\|\rho\|_{L^{\infty}\left(\Omega \times\left[0, T_{*}^{\prime}\right]\right)}^{\alpha} \cdot\left\|\rho u_{y}\right\|_{L^{\infty}\left(\Omega \times\left[0, T_{*}^{\prime}\right]\right)} \leq C\left(T_{*}^{\prime}\right) .
$$

Making use of Eq. (4.7), (4.30) and (4.31), one can obtain

$$
\begin{gathered}
\|\rho(t)-\rho(s)\|_{L^{2}(\Omega)} \leq \int_{s}^{t}\left\|\rho_{t}(\tau)\right\|_{L^{2}(\Omega)} d \tau=\left\|\int_{s}^{t} \rho^{2} u_{y}(\tau)\right\|_{L^{2}(\Omega)} d \tau \\
\leq C(t-s)\|\rho\|_{L^{\infty}\left(\Omega \times\left[0, T_{*}^{\prime}\right]\right)} \cdot\left\|\rho u_{y}\right\|_{L^{\infty}\left(\Omega \times\left[0, T_{*}^{\prime}\right]\right)} \leq C\left(T_{*}^{\prime}\right)|t-s|, \\
\|u(t)-u(s)\|_{L^{2}(\Omega)} \leq\left\|\int_{s}^{t} u_{t}(\tau) d \tau\right\|_{L^{2}(\Omega)} \leq C \int_{s}^{t}\left\|u_{t}\right\|_{L^{2}(\Omega)} \leq C\left(T_{*}^{\prime}\right)|t-s|,
\end{gathered}
$$

and

$$
\begin{aligned}
& \left\|\rho^{1+\alpha} u_{y}(t)-\rho^{1+\alpha} u_{y}(s)\right\|_{L^{2}(\Omega)} \leq\left\|\int_{s}^{t}\left(\rho^{1+\alpha} u_{y}(t)\right)_{t} d \tau\right\|_{L^{2}(\Omega)} \\
\leq & C\left(\left\|\int_{s}^{t} \rho_{t} \rho^{\alpha} u_{y}(\tau)\right\|_{L^{2}(\Omega)}+\left\|\int_{s}^{t} \rho^{1+\alpha} u_{y t}(\tau) d \tau\right\|_{L^{2}(\Omega)}\right) \\
\leq & C\left(\left\|\int_{s}^{t} \rho^{2+\alpha} u_{y}^{2}(\tau)\right\|_{L^{2}(\Omega)}+\left\|\int_{s}^{t} \rho^{1+\alpha} u_{y t}(\tau) d \tau\right\|_{L^{2}(\Omega)}\right)
\end{aligned}
$$




$$
\begin{aligned}
& \leq C\left(T_{*}^{\prime}\right)\left(|t-s|\left\|\rho^{2+\alpha} u_{y}^{2}\right\|_{L^{\infty}\left(\Omega \times\left[0, T_{*}^{\prime}\right]\right)}+|t-s|^{1 / 2}\left\|\rho^{(1+\alpha) / 2} u_{y t}(\tau)\right\|_{L^{2}\left(\Omega \times\left[0, T_{*}^{\prime}\right]\right)}\right) \\
& \leq C\left(T_{*}^{\prime}\right)|t-s|^{1 / 2} .
\end{aligned}
$$

The proof is completed.

Proof of the Proposition 4.2. With the help of Lemmas 4.64.8, we are ready to prove the Proposition 4.2 .

(1) Existence of weak solution for short time. We only deal with the case for the Dirichlet boundary condition and one point vacuum state in the initial data, the case of periodic boundary and one point vacuum state in initial data can be done in a similar way. Once the a-priori estimates are established as in Lemmas 4.6 4.9, we are able to prove the Proposition 4.2. First of all, we construct a sequence of approximate solutions by modifying the finite difference scheme used in [33,40,43]. Without the loss of generality, we assume

$$
y_{0}=\frac{1}{2}, \quad \text { namely, } \quad \rho_{0}\left(\frac{1}{2}\right)=0 .
$$

For any given positive integer $N=2 k+1$ with $k \geq 0$ an integer, let $h=1 / N$. Consider the system of $2 N$ ordinary differential equations

$$
\left\{\begin{array}{l}
\frac{d}{d t} \rho_{2 n+1}^{h}+\left(\rho_{2 n+1}^{h}\right)^{2} \frac{u_{2 n+2}^{h}-u_{2 n}^{h}}{h}=0 \\
\frac{d}{d t} u_{2 n}^{h}+\frac{\left(\rho_{2 n+1}^{h}\right)^{\gamma}-\left(\rho_{2 n-1}^{h}\right)^{\gamma}}{h} \\
\quad=\frac{1}{h}\left\{\left(\rho_{2 n+1}^{h}\right)^{1+\alpha}\left(u_{2 n+2}^{h}-u_{2 n}^{h}\right) / h-\left(\rho_{2 n-1}^{h}\right)^{1+\alpha}\left(u_{2 n}^{h}-u_{2 n-2}^{h}\right) / h\right\}
\end{array}\right.
$$

with the boundary condition and point vacuum

$$
u_{0}^{h}(t)=u_{2 N}^{h}(t)=0, \quad \rho_{2 k+1}(t)=0,
$$

and initial data

$$
\rho_{2 n+1}^{h}(0)=\rho_{0}\left((2 n+1) \frac{h}{2}\right) \quad u_{2 n}^{h}(0)=u_{0}\left((2 n) \frac{h}{2}\right)
$$

where $n=1,2,3, \ldots, N$. Here, we also assume

$$
\rho_{2 N+1}^{h}(t)=\rho_{2 N-1}^{h}(t), \quad \rho_{1}^{h}(t)=\rho_{-1}^{h}(t), \quad u_{2 N+2}^{h}(t)=u_{-2}^{h}(t)=0
$$

which is consistent with the boundary condition and the fact that density is continuous and non-zero at the left boundary.

By applying the idea as in $[32,43]$ and the similar arguments mentioned above, we can obtain the following uniform (w.r.t. $h$ ) a-priori estimates about the solutions $\left(\rho_{2 n+1}, u_{2 n}\right)$ (here and below we omit the symbol $h$ for simplicity) of (4.57)-(4.59) similar to Lemmas 4.6 4.9. Details will be omitted (the reader can refer to [32,43] for similar arguments in details). 
Lemma 4.10 Let $\left(\rho_{2 n+1}, u_{2 n}\right)$ be the solution of (4.57)-4.59). Then, it holds

$$
\begin{gathered}
\sum_{n=0}^{N}\left(\frac{1}{2} u_{2 n}^{2}(t)+\pi\left(\rho_{2 n+1}(t)\right)\right) h+\int_{0}^{t} \sum_{n=1}^{N} \rho_{2 n+1}^{1+\alpha}\left(\frac{u_{2 n+2}(s)-u_{2 n}(s)}{h}\right)^{2} h d s \\
=\sum_{n=0}^{N}\left(\frac{1}{2} u_{2 n}^{2}(0)+\pi\left(\rho_{2 n+1}(0)\right)\right) h, \\
\sum_{n=0}^{N-1} \rho_{2 n+1}^{-1}(t) h=\sum_{n=0}^{N-1} \rho_{2 n+1}^{-1}(0) h .
\end{gathered}
$$

It follows from Lemma 4.10 and the standard theory of ordinary differential equations that there exists a global solution $\left(\rho_{2 n+1}, u_{2 n}\right)$ to (4.57)-(4.59) for any fixed positive $N$ and $h$. Furthermore, the following properties hold:

Lemma 4.11 Let $\left(\rho_{2 n+1}, u_{2 n}\right)$ with $n=0,2,3, \ldots, N-1$ with $N=2 k+1$ be the solution of 4.57) 4.59). Then, it holds for $n \geq k+1$ that

$$
\begin{gathered}
\rho_{2 n+1}^{\alpha}(t)=\rho_{2 n+1}^{\alpha}(0)-\alpha \int_{0}^{t} \sum_{j=k+1}^{n} \frac{d}{d t} u_{2 j}(s) h d s-\alpha \int_{0}^{t} \rho_{2 n+1}^{\gamma}(s) d s, \\
\rho_{2 n+1}^{1+\alpha}(t) \frac{u_{2 n+2}(t)-u_{2 n}(t)}{h}=\sum_{j=k+1}^{n} \frac{d}{d t} u_{2 j}(t) h+\rho_{2 n+1}^{\gamma},
\end{gathered}
$$

and for $n \leq k$ that

$$
\begin{gathered}
\rho_{2 n-1}^{\alpha}(t)=\rho_{2 n-1}^{\alpha}(0)-\alpha \int_{0}^{t} \sum_{j=n}^{k} \frac{d}{d t} u_{2 j}(s) h d s-\alpha \int_{0}^{t} \rho_{2 n-1}^{\gamma}(s) d s, \\
\rho_{2 n-1}^{1+\alpha}(t) \frac{u_{2 n}(t)-u_{2 n-2}(t)}{h}=-\sum_{j=n}^{k} \frac{d}{d t} u_{2 j}(t) h+\rho_{2 n-1}^{\gamma} .
\end{gathered}
$$

Lemma 4.12 Under the assumptions of Proposition 4.2, there is a short time $T_{*}^{\prime}>0$ so that it holds for $n=0,1,2, \ldots, N$ that

$$
\begin{gathered}
\rho_{2 n+1}(t)+\sum_{n=1}^{N}\left(\frac{\rho_{2 n+1}^{\alpha}(t)-\rho_{2 n-1}^{\alpha}(t)}{h}\right)^{2} h+\left|\rho_{2 n+1}(t) \frac{u_{2 n+2}(t)-u_{2 n}(t)}{h}\right| \leq C\left(T_{*}^{\prime}\right), \\
a_{-}\left|\frac{1}{2}(2 n+1) h-\frac{1}{2}\right|^{\beta} \leq \rho_{2 n}(t) \leq a_{+}\left|\frac{1}{2}(2 n+1) h-\frac{1}{2}\right|^{\beta},
\end{gathered}
$$

and for $m=1$ or $n$ that

$$
\left.\sum_{j=0}^{N}\left(\frac{d}{d t} u_{2 j}(t)\right)^{2 m} h+\int_{0}^{t} \sum_{j=0}^{N} \rho_{2 j+1}^{1+\alpha}(s)\left(\frac{d}{d t} u_{2 j}(s)\right)^{2 m-2}\left(\frac{\frac{d}{d t} u_{2 n}(s)-\frac{d}{d t} u_{2 n-2}(s)}{h}\right)^{2}\right) h \leq C\left(T_{*}^{\prime}\right) .
$$

Here $C\left(T_{*}^{\prime}\right)>0$ and $a_{ \pm}>0$ are constants. 
Lemma 4.13 Under the assumptions of Proposition 4.2 and Lemma 4.12, it holds for $t \in\left[0, T_{*}^{\prime}\right]$ that

$$
\begin{gathered}
\left|\rho_{2 n+1}^{1+\alpha}(t) \frac{u_{2 n+2}(t)-u_{2 n}(t)}{h}\right| \leq C\left(T_{*}^{\prime}\right), \\
\left|u_{2 n}(t)\right|+\sum_{n=1}^{N}\left|u_{2 n}(t)-u_{2 n-2}(t)\right| \leq C\left(T_{*}^{\prime}\right), \\
\sum_{n=1}^{N}\left|\left(\rho_{2 n+1}\right)^{1+\alpha}\left(u_{2 n+2}-u_{2 n}\right) / h-\left(\rho_{2 n-1}\right)^{1+\alpha}\left(u_{2 n}-u_{2 n-2}\right) / h\right| \leq C\left(T_{*}^{\prime}\right), \\
\sum_{n=1}^{N}\left|\rho_{2 n}(t)-\rho_{2 n}(s)\right|^{2} h+\sum_{n=1}^{N}\left|u_{2 n-1}(t)-u_{2 n-3}\right|^{2} h \leq C\left(T_{*}^{\prime}\right)|t-s|^{2}, \\
\sum_{n=1}^{N}\left|\left(\rho_{2 n}\right)^{1+\alpha}(t) \frac{u_{2 n+1}(t)-u_{2 n-1}(t)}{h}-\left(\rho_{2 n-2}\right)^{1+\alpha}(s) \frac{u_{2 n-1}(s)-u_{2 n-3}(s)}{h}\right|^{2} h \leq C\left(T_{*}^{\prime}\right)|t-s| .
\end{gathered}
$$

With the help of Lemmas 4.10 4.13, we can define the sequence of approximate solutions $\left(\rho_{h}, u_{h}\right)$ on the domain $\Omega \times\left[0, T_{*}^{\prime}\right]$ as

$$
\left\{\begin{array}{l}
\rho_{h}(y, t)=\rho_{2 n+1}(t) \\
u_{h}(y, t)=\frac{1}{h}\left[\left(y-\left(n-\frac{1}{2}\right) h\right) u_{2 n+1}(t)+\left(\left(n+\frac{1}{2}\right) h-y\right) u_{2 n-1}(t)\right]
\end{array}\right.
$$

for $y \in\left((2 n) \frac{1}{2} h,(2 n+2) \frac{1}{2} h\right)$. It can be verified that the following properties hold for the approximate solutions

$$
\partial_{y} u_{h}(y, t)=\frac{u_{2 n+1}(t)-u_{2 n-1}(t)}{h}
$$

and

$$
\left\{\begin{array}{l}
a_{-}\left|y-y_{0}\right|^{\beta} \leq \rho_{h}(y, t) \leq a_{+}\left|y-y_{0}\right|^{\beta} \\
\left|u_{h}(y, t)\right| \leq C\left(T_{*}^{\prime}\right), \quad\left|\rho_{h}(y, t) \partial_{x} u_{h}(y, t)\right| \leq C\left(T_{*}^{\prime}\right),
\end{array}\right.
$$

for $(y, t) \in \Omega \times\left[0, T_{*}^{\prime}\right]$. Then, the existence of weak solution for short time $t \in\left[0, t_{*}^{\prime}\right]$ follows from Helly's theorem, the diagonal process together with Lebesgue's theorem, and the a-priori estimates (see, for instance, [33]). The details are omitted.

(2) Uniqueness of weak solution for short time. We prove the uniqueness of weak solutions for $\alpha \in\left(\frac{1}{2}, \gamma\right)$. Without loss of generality, only the case for the Dirichlet boundary condition (4.10) will be studied. Let $\left(\rho_{1}, u_{1}\right)$ and $\left(\rho_{2}, u_{2}\right)$ be two weak solutions of Eq. (4.7)-(4.10) satisfying Lemma 4.6 4.9, Denote

$$
n=\rho_{1}-\rho_{2}, \quad \psi=u_{1}-u_{2}, \quad(x, t) \in(0,1) \times[0, T] .
$$

Obviously, the new unknown $(n, \psi)$ with $\psi(0, t)=\psi(1, t)=n\left(y_{0}, t\right)=0$ satisfies

$$
\left(\frac{n}{\rho_{1} \rho_{2}}\right)_{t}+\psi_{y}=0, \quad y \neq y_{0}
$$




$$
\psi_{t}+\left(\rho_{1}^{\gamma}-\rho_{2}^{\gamma}\right)_{y}-\left(\rho_{1}^{1+\alpha} \psi_{y}\right)_{y}-\left(\left(\rho_{1}^{1+\alpha}-\rho_{2}^{1+\alpha}\right) u_{2 y}\right)_{y}=0
$$

for $(y, t) \in(0,1) \times\left(0, T_{*}\right]$ with zero initial data

$$
(n(y, 0), \psi(y, 0))=(0,0), \quad y \in(0,1) .
$$

Take inner product between $\rho_{1}^{\alpha} \rho_{2}^{-1} n$ and (4.60) over $\left[0, y_{0}\right) \cup\left(y_{0}, 1\right]$ to obtain

$$
\begin{aligned}
& \frac{d}{d t} \oint \rho_{1}^{-1+\alpha} \rho_{2}^{-2} n^{2} d y=-(1+\alpha) \oint u_{1 y} \rho_{1}^{\alpha} \rho_{2}^{-2} n^{2} d y-2 \oint \rho_{1}^{\alpha} \rho_{2}^{-1} n \psi_{y} d y \\
& \leq\left(C\left\|\rho_{1} u_{1 y}\right\|_{L^{\infty}}+4\right) \oint \rho_{1}^{-1+\alpha} \rho_{2}^{-2} n^{2} d y+\frac{1}{4} \oint \rho_{1}^{1+\alpha} \psi_{y}^{2} d y \\
& \leq C\left(T_{*}\right) \oint \rho_{1}^{-1+\alpha} \rho_{2}^{-2} n^{2} d y+\frac{1}{4} \oint \rho_{1}^{1+\alpha} \psi_{y}^{2} d y,
\end{aligned}
$$

where $\oint f d x=: \int_{0}^{y_{0}} f d x+\int_{y_{0}}^{1} f d x$. Taking inner product between $\psi$ and (4.61) over $\Omega$, and noting that $\psi(0, t)=\psi(1, t)=n\left(y_{0}, t\right)=0$ and $\rho_{i}^{1+\alpha} u_{i}\left(y_{0}, t\right)=0, i=1,2$, we have

$$
\begin{aligned}
\frac{1}{2} \frac{d}{d t}\|\psi(t)\|^{2}+\oint \rho_{1}^{1+\alpha} \psi_{y}^{2} d y=\oint\left(\rho_{1}^{\gamma}-\rho_{2}^{\gamma}\right) \psi_{y} d y-\oint\left(\rho_{1}^{1+\alpha}-\rho_{2}^{1+\alpha}\right) u_{2 y} \psi_{y} d y \\
=\oint \frac{\rho_{1}^{\gamma}-\rho_{2}^{\gamma}}{\rho_{1}-\rho_{2}} n \psi_{y} d y-\oint \frac{\rho_{1}^{1+\alpha}-\rho_{2}^{1+\alpha}}{\rho_{1}-\rho_{2}} n u_{2 y} \psi_{y} d y \\
\leq C \oint\left(\frac{\rho_{1}^{\gamma}-\rho_{2}^{\gamma}}{\rho_{1}-\rho_{2}}\right)^{2} \rho_{1}^{-1-\alpha} n^{2} d y+\frac{1}{8} \oint \rho_{1}^{1+\alpha} \psi_{y}^{2} d y \\
\quad+4 \oint\left(\frac{\rho_{1}^{1+\alpha}-\rho_{2}^{1+\alpha}}{\rho_{1}-\rho_{2}}\right)^{2} \rho_{1}^{-1-\alpha} n^{2} u_{2 y}^{2} d y+\frac{1}{8} \oint \rho_{1}^{1+\alpha} \psi_{y}^{2} d y \\
\leq \frac{1}{4} \int \rho_{1}^{1+\alpha} \psi_{y}^{2} d y+C \oint \rho_{1}^{-1+\alpha} \rho_{2}^{-2} n^{2} d y .
\end{aligned}
$$

Summing up the two differential inequalities (4.63) and (4.64) leads to

$$
\begin{aligned}
& \frac{d}{d t}\|\psi(t)\|^{2}+\frac{d}{d t} \oint \rho_{1}^{-1+\alpha} \rho_{2}^{-2} n(t) d x+\oint \rho_{1}^{1+\alpha} \psi_{y}^{2} d y \\
\leq & C\left(T_{*}\right) \oint \rho_{1}^{-1+\alpha} \rho_{2}^{-2} n^{2} d y
\end{aligned}
$$

which, together with the initial data (4.62) and the Grönwall's lemma, gives rise to

$$
\left\|\psi_{y}(t)\right\|^{2}+\oint \rho_{1}^{-1+\alpha} \rho_{2}^{-2} n^{2}(x, t) d x \equiv 0, \quad t \in[0, T]
$$

This together with $\rho_{1}\left(y_{0}, t\right)=\rho_{2}\left(y_{0}, t\right)=0$ implies the uniqueness of weak solution

$$
\left(\rho_{1}, u_{1}\right) \equiv\left(\rho_{2}, u_{2}\right)
$$


The proof of Proposition 4.2 is completed.

Proof of Proposition 4.1. With the help of Propositions 4.24.4 and the inverse transformation from the Lagrangian coordinates to the Eulerian coordinates, we are able to prove the Proposition 4.1. Since the case of periodic boundary conditions with one point vacuum state in the initial data can be dealt with in a similar framework, we only deal with the IBVP problem for the compressible Navier-Stokes equations (2.1)(2.3) for the case of the Dirichlet boundary condition (2.6) with one point vacuum in the regular initial data (2.18) $-(2.19)$.

First, one can check easily that the IBVP problem (2.1) $-(2.3),(2.6)$, and (2.18) (2.19) in the Eulerian coordinates is equivalent to the corresponding IBVP problem in the Lagrangian coordinates for the equations (4.7)-(4.9) with the Dirichlet boundary conditions (4.10) and (4.12) through the coordinate transformation

$$
y=\int_{0}^{x} \rho(z, t) d z, \quad x \in(0,1), \quad t \geq 0 .
$$

Note that $y \in[0,1]$ due to the conservation of mass. More importantly, the case of one point vacuum state in the initial data $(2.18)-(2.19)$ is reformulated into the corresponding case (4.12) with $y_{0}=\int_{0}^{x_{0}} \rho_{0}(z) d z$ and $\beta=\frac{\sigma}{1+\sigma}$. It is easy to verify that all the assumptions of Proposition 4.2 are satisfied. Then Proposition 4.2 gives the existence and uniqueness of weak solution $(\tilde{\rho}, \tilde{u})$ satisfying (4.13)-(4.18) in the Lagrangian coordinate to the compressible Navier-Stokes equations (4.7)-(4.9) with the Dirichlet boundary conditions (4.10) and one point vacuum state in the initial data. In particular,

$$
\begin{gathered}
\tilde{\rho}, \tilde{u} \in C^{0}\left(\left[0, T_{*}^{\prime}\right] ; C^{0}(\bar{\Omega})\right), \quad \tilde{\rho} \tilde{u}_{y}(t) \in L^{\infty}\left(0, T_{*}^{\prime} ; C^{0}(\bar{\Omega})\right), \\
\tilde{\rho}\left(y_{0}, t\right)=0, \quad \rho(y, t)>0, y \neq y_{0} .
\end{gathered}
$$

These in turn imply, in terms of the inverse coordinate transformation of (4.68), i.e.,

$$
x=\int_{0}^{y} \tilde{\rho}^{-1}(z, t) d z, \quad y \in[0,1],
$$

the existence and uniqueness of weak solution $(\tilde{\rho}, \tilde{u})$ on the domain $(x, t) \in[0,1] \times\left[0, T_{*}^{\prime}\right]$ to the compressible Navier-Stokes equations (2.1)-(2.3) with the Dirichlet boundary conditions (2.6) and initial one point vacuum state (2.18) $-(2.19)$. Moreover, one can check that there exists one particle path $x=X_{0}(t)$ defined by

$$
\dot{X}_{0}(t)=\tilde{u}\left(X_{0}(t), t\right), t>0, \quad X_{0}(0)=x_{0}
$$

satisfying

$$
y_{0} \equiv \int_{0}^{X_{0}(t)} \tilde{\rho}(z, t) d z=\int_{0}^{x_{0}} \rho_{0}(z) d z
$$


due to the fact

$$
\frac{d}{d t} y\left(X_{0}(t), t\right)=\frac{d}{d t} \int_{0}^{X_{0}(t)} \tilde{\rho}(z, t) d z=0, \quad t \geq 0 .
$$

This, together with (4.70), gives rise to

$$
\tilde{\rho}\left(X_{0}(t), t\right)=0, \quad \text { and } \quad \tilde{\rho}(x, t)>0, x \neq X_{0}(t), t \geq 0 .
$$

Moreover, it is easy to verify that the solution $(\tilde{\rho}, \tilde{u})$ satisfies all the properties (2.23) (2.26), and particularly

$$
\left\{\begin{array}{l}
\tilde{\rho}\left(x, T_{*}\right) \geq 0 \text { on } \Omega, \quad \tilde{m}\left(., T_{*}\right)=\tilde{\rho} \tilde{u}\left(., T_{*}\right)=0, \quad \text { on }\left\{x \in \Omega \mid \rho_{0}(x)=0\right\}, \\
\tilde{\rho}\left(., T_{*}\right) \in L^{1}(\Omega) \cap L^{\gamma}(\Omega), \quad\left(\tilde{\rho}^{\alpha-1 / 2}\left(., T_{*}\right)\right)_{x} \in L^{2}(\Omega), \\
\frac{\left|\tilde{m}\left(., T_{*}\right)\right|^{2}}{\tilde{\rho}\left(x, T_{*}\right)}+\frac{\left|\tilde{m}\left(., T_{*}\right)\right|^{2+\nu}}{\tilde{\rho}^{1+\nu}\left(x, T_{*}\right)} \in L^{1}(\Omega) .
\end{array}\right.
$$

The case of periodic boundary can be treated in a similar way, we omit the details. The proof of Proposition 4.1 is completed.

\section{Vanishing of vacuum states and blow-up phenom- ena}

We shall prove that for any global entropy weak solution $(\rho, u)$ to the IBVP for compressible Navier-Stokes equations (2.1)-(2.3) together with (2.6) or (2.7), any possible vacuum state vanishes in finite time and the velocity (if definable and regular enough) blows up in finite time if vacuum state appears, for example, the density contains vacuum initially as in Theorem 2.2. The weak solution becomes a strong one after the vanishing of vacuum states and tends time-asymptotically to the non-vacuum equilibrium state exponentially.

\subsection{Vanishing of vacuum states in finite time}

Proposition 5.1 Let 2.34) hold. For any global entropy weak solution $(\rho, u)$ to the IBVP for compressible Navier-Stokes equations (2.1) -(2.2) with initial data -(2.3) and boundary condition (2.6) or (2.7) in the sense of Definition 2.5, there exists a time $T_{0}>0$ such that

$$
\inf _{x \in \bar{\Omega}} \rho(x, t)>0, \text { for all } t \geq T_{0} .
$$

Proof: To prove (5.1), we will employ an idea which has been used in [28] (see also $[22,27])$ to show the blow up behavior of both the global strong solutions to the 
IBVP for (2.1)-(2.2) with the constant viscosity and the global strong solutions to the Stokes approximation equations, with initial data containing vacuum states.

Let $T \in(0, \infty)$ be fixed. In this subsection, $C$ denotes some generic positive constant independent of $T$. First, it is noted that the total mass is conserved the total for any $t \in(0, T]$

$$
\int_{\Omega} \rho(x, t) d x=\int_{\Omega} \rho_{0}(x) d x
$$

Based on the entropy inequality (2.13), it can be deduced from (5.2) and (2.39) that for a constant $b \geq \max \{\alpha+\gamma-1,2 \alpha+1,1\}$,

$$
\sup _{0 \leq t \leq T}\left(\|\rho\|_{L^{\infty}}+\left\|\left(\rho^{b}\right)_{x}\right\|_{L^{2}}\right)+\int_{0}^{T}\left\|\left(\rho^{b}\right)_{x}\right\|_{L^{2}}^{2} d t \leq C .
$$

I t will be shown below that

$$
g(t) \triangleq\left\|\left(\rho^{b}-\overline{\rho^{b}}\right)(\cdot, t)\right\|_{L^{4}(\Omega)}^{4} \rightarrow 0 \text { as } t \rightarrow \infty
$$

where

$$
\overline{\rho^{b}}(t)=\frac{1}{|\Omega|} \int_{\Omega} \rho^{b}(x, t) d x .
$$

Now, we assume that (5.4) holds, and continue the proof of Proposition 5.1. In fact, the inequality (5.3) and the Poincáre-Sobolev inequality imply that

$$
\begin{aligned}
\left\|\left(\rho^{b}-\overline{\rho^{b}}\right)(\cdot, t)\right\|_{C(\bar{\Omega})} & \leq C\left\|\left(\rho^{b}-\overline{\rho^{b}}\right)(\cdot, t)\right\|_{L^{4}(\Omega)}^{2 / 3}\left\|\left(\rho^{b}\right)_{x}(\cdot, t)\right\|_{L^{2}}^{1 / 3} \\
& \leq C\left\|\left(\rho^{b}-\overline{\rho^{b}}\right)(\cdot, t)\right\|_{L^{4}(\Omega)}^{2 / 3} \rightarrow 0 \text {, as } t \rightarrow \infty .
\end{aligned}
$$

This suffices to finish the proof of Proposition 5.1 due to the following simple fact

$$
\overline{\rho^{b}}(t) \geq \bar{\rho}^{b}(t) \equiv{\overline{\rho_{0}}}^{b}=1, \text { for any } t \geq 0 \text {. }
$$

It remains to prove (5.4). First, it follows directly from (5.3) and the PoincáreSobolev inequality that

$$
\int_{0}^{T} g(t) d t \leq C \sup _{0 \leq t \leq T}\left\|\rho^{b}-\overline{\rho^{b}}\right\|_{L^{\infty}}^{2} \int_{0}^{T}\left\|\left(\rho^{b}\right)_{x}\right\|_{L^{2}}^{2} d t \leq C .
$$

Next, we prove that

$$
\int_{0}^{T}\left|g^{\prime}(t)\right| d t \leq C
$$


Note that (2.9) as well as the boundary condition (2.6) or (2.7) imply that

$$
\begin{aligned}
g^{\prime}(t) & =4 b\left\langle\left(\rho^{b}-\overline{\rho^{b}}\right)^{3} \rho^{b-1}, \rho_{t}\right\rangle_{H^{1} \times H^{-1}}-4\left(\overline{\rho^{b}}\right)_{t} \int_{\Omega}\left(\rho^{b}-\overline{\rho^{b}}\right)^{3} d x \\
& =-4 b \int_{\Omega}\left(\left(\rho^{b}-\overline{\rho^{b}}\right)^{3} \rho^{b-1}\right)_{x} \sqrt{\rho} \sqrt{\rho} u d x-4\left(\overline{\rho^{b}}\right)_{t} \int_{\Omega}\left(\rho^{b}-\overline{\rho^{b}}\right)^{3} d x \\
& \triangleq I_{1}+I_{2} .
\end{aligned}
$$

It follows from (5.3) and (2.13) that

$$
\begin{aligned}
\int_{0}^{T}\left|I_{1}\right| d t \leq & C \int_{0}^{T}\left|\int_{\Omega}\left(\rho^{b}-\overline{\rho^{b}}\right)^{2}\left(\rho^{b}\right)_{x} \rho^{b-1 / 2} \sqrt{\rho} u d x\right| d t \\
& +C \int_{0}^{T}\left|\int_{\Omega}\left(\rho^{b}-\overline{\rho^{b}}\right)^{3}\left(\rho^{b-1}\right)_{x} \sqrt{\rho} \sqrt{\rho} u d x\right| d t \\
\leq & C \int_{0}^{T}\left|\int_{\Omega}\left(\rho^{b}-\overline{\rho^{b}}\right)^{2}\left(\rho^{\alpha-1 / 2}\right)_{x} \rho^{b-\alpha} \sqrt{\rho} u d x\right| d t \\
\leq & C \int_{0}^{T}\left\|\left(\rho^{\alpha-1 / 2}\right)_{x}\right\|_{L^{2}}\|\sqrt{\rho} u\|_{L^{2}}\left\|\rho^{b}-\overline{\rho^{b}}\right\|_{L^{\infty}}^{2} d t \\
\leq & C \int_{0}^{T}\left\|\left(\rho^{b}\right)_{x}\right\|_{L^{2}}^{2} d t \\
\leq & C .
\end{aligned}
$$

The uniform entropy estimate (2.13), together with (2.9), gives that

$$
\begin{aligned}
\sup _{0 \leq t \leq T}\left|\frac{d}{d t} \overline{\rho^{b}}(t)\right| & =b \sup _{0 \leq t \leq T}\left|\left\langle\rho^{b-1}, \rho_{t}\right\rangle\right| \\
& =b \sup _{0 \leq t \leq T}\left|\int_{\Omega}\left(\rho^{b-1}\right)_{x} \sqrt{\rho} \sqrt{\rho} u d x\right| \\
& \leq C \sup _{0 \leq t \leq T}\left|\int_{\Omega}\left(\rho^{\alpha-1 / 2}\right)_{x} \rho^{b-\alpha} \sqrt{\rho} u d x\right| \\
& \leq C \sup _{0 \leq t \leq T}\left(\left\|\left(\rho^{\alpha-1 / 2}\right)_{x}\right\|_{L^{2}}\|\sqrt{\rho} u\|_{L^{2}}\|\rho\|_{L^{\infty}}^{b-\alpha}\right) \\
& \leq C .
\end{aligned}
$$

This together with Poincaré inequality and (5.3) yields

$$
\int_{0}^{T}\left|I_{2}\right| d t \leq C \int_{0}^{T}\left\|\rho^{b}-\overline{\rho^{b}}\right\|_{L^{\infty}}^{3} d t \leq C \int_{0}^{T}\left\|\left(\rho^{b}\right)_{x}\right\|_{L^{2}}^{2} d t \leq C .
$$

The estimate (5.7) thus follows directly from (5.8)-(5.11). Hence the desired estimate (5.4) follows from (5.6) and (5.7). The proof of Proposition 5.1 is completed. 


\subsection{Regularity and asymptotics of weak solutions for large time}

It is usually difficult to get information about the velocity field for the global entropy weak solution $(\rho, \sqrt{\rho} u)$, in the sense of Definition 2.5 to the IBVP for the Compressible Navier-Stokes equations (2.1)-(2.2) with initial data (2.3) and boundary values (2.6) or (2.7) in the appearance of vacuum states. After vacuum states vanish, however, it will be shown that the velocity field $u$ can be defined with enough regularity and the nonlinear diffusion term is represented in terms of the velocity $u$ and the density $\rho$. The momentum equation becomes a uniform parabolic equation, and the weak solution $(\rho, \sqrt{\rho} u)=(\rho, \sqrt{\rho} \cdot u)$ becomes a strong solution.

Proposition 5.1 implies that there is a time $T_{0}>0$ after which the density of the global entropy weak solution $(\rho, u)$ to the IBVP problem for (2.1)-(2.3) together with (2.6) or (2.7) is strictly positive and $(\rho, u)$ satisfies the finite entropy estimate (2.13). Consider the IBVP problem (2.1) -(2.2) again for time $t \geq T_{0}$ with data given at time $t=T_{0}$ by

$$
\rho\left(x, T_{0}\right)=\lim _{t \rightarrow T_{0}} \rho(x, t), \quad u\left(x, T_{0}\right)=\lim _{t \rightarrow T_{0}} \frac{\sqrt{\rho} u(x, t)}{\sqrt{\rho(x, t)}}
$$

and note here that away from vacuum the Dirichlet boundary condition (2.6) reduces to

$$
u(0, t)=u(1, t)=0, \quad t \geq T_{0} .
$$

We then have the regularity property of the solution for the compressible NavierStokes equations (2.1)-(2.3) with the Dirichlet boundary condition (2.6) or the periodic boundary condition (2.7) for large time.

Proposition 5.2 Under the assumptions of Theorem 2.3 , let $(\rho, \sqrt{\rho} u)$ be the global entropy weak solution to the IBVP for the compressible Navier-Stokes equations (2.1) (2.2) with initial data (2.3) and boundary value (2.6) or (2.7) in the sense of Definition 2.5. Let $T_{0}>0$ so that the global weak solution $(\rho, u)$ satisfies for two positive constants $\rho_{ \pm}$that

$$
0<\rho_{-} \leq \rho(x, t) \leq \rho_{+}, \quad \forall(x, t) \in \bar{\Omega} \times\left[T_{0}, \infty\right)
$$

Then, $(\rho, \sqrt{\rho} u)=(\rho, \sqrt{\rho} \cdot u)$ is the unique strong solution to the IBVP for the compressible Navier-Stokes equations (2.1) -(2.2) and (5.1) with the boundary condition (2.6) 2 or (2.7) for $t \geq T_{0}$. Moreover, the regularity (2.36) and the long time behavior (2.38) hold.

Proof: We only prove Proposition 5.2 for the Dirichlet case below, the periodic case can be treated similarly.

\footnotetext{
${ }^{2}$ Note here that away from vacuum the Dirichlet boundary condition (2.6) reduces to the usual one $u(0, t)=u(1, t)=0, t \geq T_{0}$.
} 
Step 1. Regularity. It follows easily from proposition 5.1 that there exist some $T_{0}$ and a constant $\rho_{-}>0$ such that for all $t \geq T_{0}$,

$$
\inf _{x \in \bar{\Omega}} \rho(x, t) \geq \rho_{-}>0
$$

which, together with (2.13), implies

$$
\rho \in L^{\infty}\left(T_{0}, T ; H^{1}(\Omega)\right)
$$

for any $T>T_{0}$. By the continuity of $\rho \in C(\bar{\Omega} \times[0, \infty))$ there exists some $\sigma>0$ small enough such that for any $t \geq T_{0}-\sigma$,

$$
\inf _{x \in \bar{\Omega}} \rho(x, t) \geq \frac{\rho_{-}}{2}>0 .
$$

This implies that one can define the velocity $u$ for any global entropy weak solution in the sense of Definition 2.5 after the vanishing of vacuum states by

$$
u=: \frac{\sqrt{\rho} u}{\sqrt{\rho}}, \quad \text { for } \quad t \geq T_{0}-\sigma .
$$

It then follows from the definition and (2.8) that

$$
u \in L^{\infty}\left(T_{0}-\sigma, T ; L^{2}(\Omega)\right)
$$

for any $T>T_{0}$. Noting that (2.12) implies that for any $\varphi(x) \in C_{0}^{\infty}(\Omega), \psi(t) \in C_{0}^{\infty}\left(T_{0}-\right.$ $\sigma, T)$,

$$
\begin{aligned}
\int_{T_{0}-\sigma}^{T} & \psi(t) \int_{\Omega} \Lambda \rho^{-\alpha} \varphi d x d t \\
= & -\int_{T_{0}-\sigma}^{T} \psi(t) \int_{\Omega} \rho^{\alpha-1 / 2} \sqrt{\rho} u\left(\rho^{-\alpha} \varphi\right)_{x} d x d t \\
& -\frac{2 \alpha}{2 \alpha-1} \int_{T_{0}-\sigma}^{T} \psi(t) \int_{\Omega}\left(\rho^{\alpha-1 / 2}\right)_{x} \sqrt{\rho} u \rho^{-\alpha} \varphi d x d t \\
= & -\int_{T_{0}-\sigma}^{T} \psi(t) \int_{\Omega} \sqrt{\rho} u \rho^{-1 / 2} \varphi_{x} d x d t \\
= & -\int_{T_{0}-\sigma}^{T} \psi(t) \int_{\Omega} u \varphi_{x} d x d t
\end{aligned}
$$

we can define the spatial derivative of velocity and, together with (2.13), its regularity as

$$
u_{x}=\frac{\Lambda}{\rho^{\alpha}} \in L^{2}\left(\Omega \times\left(T_{0}-\sigma, T\right)\right) .
$$

In terms of (5.6), (5.7) and (2.9) we are also able to justify the Dirichlet boundary condition (2.6) for the velocity $u$

$$
u(0, t)=u(1, t)=0, \text { for any } t \geq T_{0}-\sigma .
$$


Thus, (5.6), (5.7) and (5.8) show

$$
u \in L^{2}\left(T_{0}-\sigma, T ; H_{0}^{1}(\Omega)\right) \cap L^{\infty}\left(T_{0}-\sigma, T ; L^{2}(\Omega)\right)
$$

for the case of the Dirichlet boundary conditions. Note here that $u \in L^{2}\left(T_{0}-\right.$ $\left.\sigma, T ; H_{\text {per }}^{1}(\Omega)\right) \cap L^{\infty}\left(T_{0}-\sigma, T ; L^{2}(\Omega)\right)$ in the case of periodic boundary conditions. We thus obtain from (2.9) and (2.10) that the solution $(\rho, u)$ satisfies

$$
\rho_{t}+(\rho u)_{x}=0 \quad \text { a.e. in } \quad \Omega \times\left(T_{0}-\sigma, T\right),
$$

and

$$
\int_{T_{0}-\sigma}^{T} \int_{\Omega} \rho u \varphi_{t} d x d t+\int_{T_{0}-\sigma}^{T} \int_{\Omega}\left(\rho u^{2}-\rho^{\alpha} u_{x}+\rho^{\gamma}\right) \varphi_{x} d x d t=0
$$

for any $\varphi(x, t) \in C_{0}^{\infty}\left(\Omega \times\left(T_{0}-\sigma, T\right)\right)$ for the Dirichlet case. The Eq. (5.11) can be re-written in terms of (5.9), (5.10) and (2.13) as follows

$$
\int_{T_{0}-\sigma}^{T} \int_{\Omega}\left(u \varphi_{t}-\rho^{\alpha-1} u_{x} \varphi_{x}+\left(\rho^{\alpha-2} \rho_{x}-u\right) u_{x} \varphi\right) d x d t=\int_{T_{0}-\sigma}^{T} \int_{\Omega} \gamma \rho^{\gamma-2} \rho_{x} \varphi d x d t
$$

for any $\varphi(x, t) \in C_{0}^{\infty}\left(\Omega \times\left(T_{0}-\sigma, T\right)\right)$ for the Dirichlet case.

Noticing that $\rho^{\alpha-2} \rho_{x}-u \in L^{\infty}\left(T_{0}-\sigma, T ; L^{2}(\Omega)\right)$ due to (2.8), (5.4) and (5.6), and using standard regularity results for linear parabolic equations (see [25]), we get that

$$
\left.u \in L^{2}\left(T_{0}, T ; H^{2}(\Omega)\right)\right) \cap H^{1}\left(T_{0}, T ; L^{2}(\Omega)\right)
$$

for the Dirichlet case. It is noted here that $u \in L^{2}\left(T_{0}, T ; H_{\text {per }}^{2}(\Omega)\right) \cap H^{1}\left(T_{0}, T ; L^{2}(\Omega)\right)$ for the periodic case.

Step 2. Uniqueness. We shall show that if there exists another solution $(\eta, v)$ to the compressible Navier-Stokes equations (2.1)-(2.2) with the following initial data and Dirichlet boundary conditions

$$
\left\{\begin{array}{l}
(\eta, v)\left(x, T_{0}\right)=(\rho, u)\left(x, T_{0}\right) \\
v(0, t)=v(1, t)=0
\end{array}\right.
$$

such that

$$
\left\{\begin{array}{l}
\rho_{-} \leq \eta \in L^{\infty}\left(T_{0}, T ; H^{1}(\Omega)\right), \\
v \in L^{\infty}\left(T_{0}, T ; H^{1}(\Omega)\right) \cap L^{2}\left(T_{0}, T ; H^{2}(\Omega)\right) \cap H^{1}\left(T_{0}, T ; L^{2}(\Omega)\right),
\end{array}\right.
$$

then

$$
\rho=\eta, \quad u=v \quad \text { a.e. in } \Omega \times\left(T_{0}, T\right) .
$$

In fact, it follows from (5.10), (5.11) and (5.13) that

$$
\frac{1}{2} \int_{\Omega} \rho u^{2} d x+\int_{T_{0}}^{t} \int_{\Omega} \rho^{\alpha} u_{x}^{2} d x d s-\int_{T_{0}}^{t} \int_{\Omega} \rho^{\gamma} u_{x} d x d s=\frac{1}{2} \int_{\Omega} \rho u^{2}\left(x, T_{0}\right) d x
$$


for all $t \in\left(T_{0}, T\right)$, while (5.11) and (5.15) imply that

$$
\begin{gathered}
\int_{\Omega} \rho u v d x+\int_{T_{0}}^{t} \int_{\Omega} \rho^{\alpha} u_{x} v_{x} d x d s-\int_{T_{0}}^{t} \int_{\Omega} \rho^{\gamma} v_{x} d x d s \\
=\int_{\Omega} \rho u v\left(x, T_{0}\right) d x+\int_{T_{0}}^{t} \int_{\Omega} \rho u\left(v_{t}+u v_{x}\right) d x d s
\end{gathered}
$$

for all $t \in\left(T_{0}, T\right)$. To estimate the second term on the right hand side of (5.18), we use the decomposition

$$
\rho v_{t}+\rho u v_{x}=(\rho-\eta)\left(v_{t}+v v_{x}\right)+\rho(u-v) v_{x}+\left(\eta^{\alpha} v_{x}\right)_{x}-\left(\eta^{\gamma}\right)_{x}
$$

Multiplying (5.19) by $u$ and we integrating by parts give

$$
\begin{aligned}
\int_{T_{0}}^{t} \int_{\Omega} \rho u\left(v_{t}+u v_{x}\right) d x d s & \\
= & \int_{T_{0}}^{t} \int_{\Omega}(\rho-\eta) u\left(v_{t}+v v_{x}\right) d x d s+\int_{T_{0}}^{t} \int_{\Omega} \rho u(u-v) v_{x} d x d s \\
& -\int_{T_{0}}^{t} \int_{\Omega} \eta^{\alpha} v_{x} u_{x} d x d s+\int_{T_{0}}^{t} \int_{\Omega} \eta^{\gamma} u_{x} d x d s
\end{aligned}
$$

Substituting (5.20) into (5.18) gives, for a.e. $t \in\left(T_{0}, T\right)$, that

$$
\begin{gathered}
\int_{\Omega} \rho u v d x+\int_{T_{0}}^{t} \int_{\Omega} \rho^{\alpha} u_{x} v_{x} d x d s-\int_{T_{0}}^{t} \int_{\Omega}\left(\rho^{\gamma} v_{x}+\eta^{\gamma} u_{x}\right) d x d s \\
=\int_{\Omega} \rho u v\left(x, T_{0}\right) d x+\int_{T_{0}}^{t} \int_{\Omega}(\rho-\eta) u\left(v_{t}+v v_{x}\right) d x d s \\
\quad+\int_{T_{0}}^{t} \int_{\Omega} \rho u(u-v) v_{x} d x d s-\int_{T_{0}}^{t} \int_{\Omega} \eta^{\alpha} v_{x} u_{x} d x d s
\end{gathered}
$$

Multiplying (5.19) by $v$ and integrating the result over $\Omega \times\left(T_{0}, t\right)$ lead to

$$
\begin{aligned}
\frac{1}{2} \int_{\Omega} \rho v^{2} d x= & \frac{1}{2} \int_{\Omega} \rho v^{2}\left(x, T_{0}\right) d x+\int_{T_{0}}^{t} \int_{\Omega}(\rho-\eta) v\left(v_{t}+v v_{x}\right) d x d s \\
& +\int_{T_{0}}^{t} \int_{\Omega} \rho v(u-v) v_{x} d x d s-\int_{T_{0}}^{t} \int_{\Omega} \eta^{\alpha} v_{x}^{2} d x d s \\
& +\int_{T_{0}}^{t} \int_{\Omega} \eta^{\gamma} v_{x} d x d s
\end{aligned}
$$

We obtain after adding up (5.17) and (5.22) and subtracting (5.21) that for all 
$t \in\left(T_{0}, T\right)$,

$$
\begin{aligned}
\frac{1}{2} \int_{\Omega} \rho(u-v)^{2} d x+\int_{T_{0}}^{t} \int_{\Omega} \rho^{\alpha}(u-v)_{x}^{2} d x d s \\
=\int_{T_{0}}^{t} \int_{\Omega}\left(\rho^{\alpha}-\eta^{\alpha}\right)(v-u)_{x} v_{x} d x d s+\int_{T_{0}}^{t} \int_{\Omega}(\rho-\eta)\left(v_{t}+v v_{x}\right)(v-u) d x d s \\
\quad-\int_{T_{0}}^{t} \int_{\Omega} \rho(u-v)^{2} v_{x} d x d s-\int_{T_{0}}^{t} \int_{\Omega}\left(\rho^{\gamma}-\eta^{\gamma}\right)(v-u)_{x} d x d s \\
\leq C_{\varepsilon} \int_{T_{0}}^{t}\left(\left\|\rho^{\alpha}-\eta^{\alpha}\right\|_{L^{2}}^{2}\left\|v_{x}\right\|_{L^{\infty}}^{2}+\|\rho-\eta\|_{L^{2}}^{2}\left\|v_{t}+v v_{x}\right\|_{L^{2}}^{2}+\left\|\rho^{\gamma}-\eta^{\gamma}\right\|_{L^{2}}^{2}\right) d s \\
\quad+C \int_{T_{0}}^{t}\left\|v_{x}\right\|_{L^{\infty}} \int_{\Omega} \rho(u-v)^{2} d x d s+C \varepsilon \int_{T_{0}}^{t}\|u-v\|_{H^{1}}^{2} d s .
\end{aligned}
$$

Next, we estimate the term

$$
g(t) \triangleq\|\rho-\eta\|_{L^{2}}^{2}+\left\|\rho^{\alpha}-\eta^{\alpha}\right\|_{L^{2}}^{2}+\left\|\rho^{\gamma}-\eta^{\gamma}\right\|_{L^{2}}^{2} .
$$

For any $\beta>0$, (5.15), (5.13) and (2.1) imply that

$$
\left(\rho^{\beta}-\eta^{\beta}\right)_{t}+v\left(\rho^{\beta}-\eta^{\beta}\right)_{x}+(u-v)\left(\rho^{\beta}\right)_{x}+\beta \rho^{\beta}(u-v)_{x}+\beta\left(\rho^{\beta}-\eta^{\beta}\right) v_{x}=0 .
$$

One can derive from this that

$$
\begin{aligned}
\left(\left\|\rho^{\beta}-\eta^{\beta}\right\|_{L^{2}}^{2}\right)_{t} \leq & \left\|\rho^{\beta}-\eta^{\beta}\right\|_{L^{2}}^{2}\left(C\left\|v_{x}\right\|_{L^{\infty}}+C_{\varepsilon}\left\|\rho_{x}\right\|_{L^{2}}^{2}+C_{\varepsilon}\right) \\
& +\varepsilon\|u-v\|_{L^{\infty}}^{2}+\varepsilon\left\|(u-v)_{x}\right\|_{L^{2}}^{2} .
\end{aligned}
$$

Thus, since $g\left(T_{0}\right)=0$, we obtain from (5.25) with $\beta=1, \alpha, \gamma$ respectively that

$$
g(t) \leq \int_{T_{0}}^{t} g(s)\left(C\left\|v_{x}\right\|_{L^{\infty}}+C_{\varepsilon}\left\|\rho_{x}\right\|_{L^{2}}^{2}+C_{\varepsilon}\right) d s+C \varepsilon \int_{T_{0}}^{t}\|u-v\|_{H^{1}}^{2} d s .
$$

Now (5.16) is a consequence from (5.23), (5.26), (5.15), (5.5) and (5.4). The proof of large time convergence (2.38) follows directly from the standard arguments (see [44] for instance) with the help of entropy inequality (2.13). The proof of Proposition 5.2 is completed.

\subsection{Finite time blow-up}

In this subsection, we shall prove the Theorem 2.4 about the finite time blowup phenomena as an immediate consequence of Theorem 2.3. Propositions 5.1 and Proposition 5.2. 
Proof of Theorem 2.4. We will prove (2.42) only. The proof of (2.43) is similar. If (2.42) fails, then there exists a fixed constant $\eta>0$, such that

$$
\int_{T_{1}}^{T_{1}+\eta}\left\|u_{x}\right\|_{L^{\infty}} d s<\infty
$$

For any $(x, t) \in \bar{\Omega} \times\left(T_{1}, T_{1}+\eta\right]$, the particle path $x(s)=X(s ; t, x) \operatorname{through}(x, t)$ is given by

$$
\begin{cases}\frac{\partial}{\partial s} X(s ; t, x)=u(X(s ; t, x), s), & T_{1} \leq s<t \leq T_{1}+\eta, \\ X(t ; t, x)=x, & T_{1} \leq t \leq T_{1}+\eta, x \in \bar{\Omega}\end{cases}
$$

which is well-defined due to (5.27) and (2.36). Consequently, one obtains via a standard argument from the transport equation (2.1)

$$
\rho(x, t)=\rho\left(X\left(T_{1} ; t, x\right), T_{1}\right) \exp \left\{-\left.\int_{T_{1}}^{t} u_{y}(y, s)\right|_{y=X(s ; t, x)} d s\right\}
$$

for any $(x, t) \in \bar{\Omega} \times\left(T_{1}, T_{1}+\eta\right]$. On the other hand, it follows from (5.27) and (5.28) that for any $x \in \bar{\Omega}$ there exists a trajectory $x=x(t) \in \bar{\Omega}$ for $t \in\left[T_{1}, T_{1}+\eta\right]$ so that $X\left(T_{1} ; t, x(t)\right)=x$. In particular, there exists a trajectory $x=x_{1}(t) \in \bar{\Omega}$ for $t \in\left[T_{1}, T_{1}+\eta\right]$ so that $X\left(T_{1} ; t, x(t)\right)=x_{1}$ with $\left(x_{1}, T_{1}\right)$ determined by (2.40), namely, $\rho\left(x_{1}, T_{1}\right)=0$. Thus, due to (5.29), we deduce from (5.27) that

$$
\rho\left(x_{1}(t), t\right) \equiv 0 \quad \text { for all } \quad t \in\left(T_{1}, T_{1}+\eta\right]
$$

which contradicts (2.40). Thus, the blowup phenomena (2.42) happens. The proof of the Theorem 2.4 is completed.

Acknowledgements The authors would like to thank the referee for informing them the recent published references $[4,5,39]$ and the helpful comments on the manuscript. They also would like to thank Prof. Didier Bresch for his interests in this work and helpful discussions about the BD entropy estimates he and his collaborators introduced.

The main part of this research was done when H.Li and J. Li were visiting the Institute of Mathematical Sciences (IMS) of The Chinese University of Hong Kong. The financial supports form the IMS and the hospitality of the staff at the IMS are appreciated greatly. The research of H. Li is partially supported by Beijing Nova Program, NNSFC No.10431060, the NCET support of the Ministry of Education of China, the institute of Mathematics and interdisciplinary Science at CNU, the Re Shi Bu Ke Ji Ze You program, and Zheng Ge Ru foundation. The research of J. Li is partially supported by the JSPS Research Fellowship for foreign researchers, NNSFC No.10601059. The research of J. Li and Xin are partially supported by Hong Kong RGC Earmarked Research Grants CUHK4028/04 and 4040/02, and Zheng Ge Ru Foundation. 


\section{References}

[1] Balian, R. From microphysics to macrophysics. Springer, Berlin, 1982.

[2] Bresch, D.; Desjardins, B. Existence of global weak solutions for a 2D viscous shallow water equations and convergence to the quasi-geostrophic model, Comm. Math. Phys. 238 (2003), 211-223.

[3] Bresch, D.; Desjardins, B. Some diffusive capillary models for Korteweg type. C. R. Mecanique 331, 2003.

[4] Bresch, D.; Desjardins, B. On the construction of approximate solutions for the $2 \mathrm{D}$ viscous shallow water model and for compressible Navier-Stokes models. $J$. Math. Pures Appl. 86 (2006), no. 4, 362-368.

[5] Bresch D.; Desjardins B.; Gérard-Varet D. On compressible Navier-Stokes equations with density dependent viscosities in bounded domains. J. Math. Pures Appl. 87 (2007), no. 2, 227-235.

[6] Bresch, D.; Desjardins, B. On the existence of global weak solutions to the NavierStokes equations for viscous compressible and heat conducting fluids. J. Math. Pures Appl. 87 (2007), no. 1, 57-90.

[7] Bresch, D.; Desjardins, B.; Lin, C.-K. On some compressible fluid models: Korteweg, lubrication, and shallow water systems. Comm. Partial Differential Equations 28 (2003), 843-868.

[8] Bresch D.; Desjardins B.; Métivier G. Recent Mathematical Results and Open Problems About Shallow Water Equations, Birkauser 2007.

[9] Cho, Y. ; Choe, H. J. ; Kim, H. Unique solvability of the initial boundary value problems for compressible viscous fluids. J. Math. Pures Appl. 83 (2004), no. 2, 243-275.

[10] Choe, H.J.; Kim, H. Strong solutions of the Navier-Stokes equations for isentropic compressible fluids. J. Differential Equations 190 (2003), no. 2, 504-523.

[11] Danchin, R. Global existence in critical spaces for compressible Navier-Stokes equations. Invent. Math. 141 (2000), 579-614.

[12] Fang, D.; Zhang, T. Compressible Navier-Stokes equations with vacuum state in one dimension. Comm. Pure Appl. Anal. 3 (2004), 675-694.

[13] Feireisl, E.; Novotny, A.; Petzeltová, H. On the existence of globally defined weak solutions to the Navier-Stokes equations. J. Math. Fluid Mech. 3 (2001), no. 4, 358-392.

[14] Gerbeau, J.-F.; Perthame, B. Derivation of viscous Saint-Venant system for laminar shallow water; numerical validation. Discrete Contin. Dyn. Syst. Ser. B1, (2001), 89-102. 
[15] Guo, Z.-H.; Jiang, S.; Xie, F. Global existence and asymptotic behavior of weak solutions to the $1 D$ compressible Navier-Stokes equations with degenerate viscosity coefficient, submitted for publication 2007.

[16] Guo, Z.-H.; Jiu, Q.-S.; Xin, Z. Spherically symmetric isentropic compressible flows with density-dependent viscosity coefficients, to appear in SIAM Journal on Mathematical Analysis, (2007).

[17] Guo, Z.-H.; Zhu, C.-J., Global weak solutions and asymptotic behavior to 1D compressible Navier-Stokes equations with density-dependent viscosity and vacuum, submitted for publication 2007

[18] Hoff, D. Global existence for 1D, compressible, isentropic Navier-Stokes equations with large initial data. Trans. Amer. Math. Soc. 303 (1987), no. 1, 169-181.

[19] Hoff, D. Strong convergence to global solutions for multidimensional flows of compressible, viscous fluids with polytropic equations of state and discontinuous initial data. Arch. Rational Mech. Anal. 132 (1995), 1-14.

[20] Hoff, D.; Serre, D. The failure of continuous dependence on initial data for the Navier-Stokes equations of compressible flow. SIAM J. Appl. Math. 51 (1991), no. $4,887-898$.

[21] Hoff, D.; Smoller, J., Non-formation of vacuum states for compressible NavierStokes equations. Comm. Math. Phys. 216 (2001), no. 2, 255-276.

[22] Huang, F.; Li, J.; Xin, Z. Convergence to equilibria and blowup behavior of global strong solutions to the Stokes approximation equations for two-dimensional compressible flows with large data. J. Math. Pures Appl. 86 (2006), no. 6, 471-491.

[23] Jiang, S.; Xin, Z.; Zhang, P. Golobal weak solutions to 1D compressible isentropic Navier-Stokes equations with density-dependent viscosity, Methods and Applications of Analysis 12 (2005), 239-251.

[24] Kazhikhov, A. V.; ShelukhinV, V. Unique global solution with respect to time of initial-boundary value problems for one-dimensional equations of a viscous gas. $J$. Appl. Math. Mech., 41 (1977), no. 2, 273-282.

[25] Ladyzenskaja, O. A.; Solonnikov, V. A.; Uraltseva, N. N. Linear and quasilinear equations of parabolic type. Translated from the Russian by S. Smith. Translations of Mathematical Monographs, Vol. 23. American Mathematical Society, Providence, R.I., 1968.

[26] Li, H.-L.; Li, J.; Xin, Z. Vanishing vacuum states and blow-up of global weak solutions for full Navier-Stokes equations, in preparation 2006.

[27] Li, J. Qualitative behavior of solutions to the compressible Navier-Stokes equations and its variants. PhD Thesis, Chinese University of Hong Kong, 2004.

[28] Li, J.; Xin Z. Some uniform estimates and blowup behavior of global strong solutions to the Stokes approximation equations for two-dimensional compressible flows. J. Differential Equations 221, (2006), 275-308. 
[29] Lions, P. L. Existence globale de solutions pour les equations de Navier-Stokes compressibles isentropiques. C. R. Acad. Sci. Paris, Sér I Math., 316 (1993), 1335-1340.

[30] Lions, P. L. Limites incompressible et acoustique pour des fluides visqueux, compressibles et isentropiques. C. R. Acad. Sci. Paris Sér. I Math. bf 317 (1993), 1197-1202.

[31] Lions, P.-L., Mathematical topics in fluid mechanics. Vol. 2. Compressible models. Oxford University Press, New York, 1998.

[32] Liu, T.-P.; Xin, Z.; Yang, T. Vacuum states for compressible flow. Discrete Contin. Dynam. Systems 4 (1998), 1-32.

[33] Luo, T.; Xin, Z.; Yang, T. Interface behavior of compressible Navier-Stokes equations with vacuum. SIAM J. Math. Anal. 31, (2000), 1175-1191.

[34] Marche, F. Derivation of a new two-dimensional shallow water model with varying topography, bottom friction and capillary effects, Eur. J. Mech. B Fluids $\mathbf{2 6}$ (2007), no. 1, 49-63.

[35] Matsumura, A.; Nishida, T. The initial value problem for the equations of motion of compressible viscous and heat-conductive fluids. Proc. Japan Acad. Ser. A Math. Sci., 55 (1979), no. 9, 337-342.

[36] Matsumura, A.; Nishida, T. The initial value problem for the equations of motion of viscous and heat-conductive gases. J. Math. Kyoto Univ., 20 (1980), no. 1, $67-104$.

[37] Matsumura, A.; Nishida, T. The initial boundary value problems for the equations of motion of compressible and heat-conductive fluids. Comm. Math. Phys. 89 (1983), 445-464.

[38] Mellet, A.; Vasseur, A. On the barotropic compressible Navier-Stokes equations, Comm. Partial Differential Equations 32 (2007), no. 1-3, 431-452.

[39] Mellet, A.; Vasseur, A. Existence and uniqueness of global strong solutions for one-dimensional compressible Navier-Stokes equations, SIAM Journal on Mathematical Analysis (2007), to appear.

[40] Nishida, T. Motion of compressible fluids in "Patterns and waves. Qualitative analysis of nonlinear differential equations", pp. 89-19, Ed. Nishida, Masayasu Mimura and Hiroshi Fujii, Kinokuniya Company Ltd., Tokyo, 1986

[41] Okada, M. Free boundary problem for the equation of one-dimensional motion of viscous gas. Japan J. Appl. Math. 6, 1989, 161-177.

[42] Okada, M.; Makino, T. Free boundary problem for the equation of spherically symmetric motion of viscous gas Japan J. Appl. Math. 10, 1993, 219-235.

[43] Okada, M.; Matsusu-Necasova, S.; Makino, T. Free boundary problem for the equation of one-dimensional motion of compressible gas with density-dependent viscosity. Ann. Univ. Ferrara Sez. VII (N.S.) 48, 2002, 1-20. 
[44] Straškraba, I; Zlotnik, A. Global properties of solutions to 1D-viscous compressible barotropic fluid equations with density dependent viscosity. Z. Angew. Math. Phys. 54 (2003), no. 4, 593-607.

[45] Salvi, R.; Straškraba I. Global existence for viscous compressible fluids and their behavior as $t \rightarrow \infty$. J. Fac. Sci. Univ. Tokyo Sect. IA, Math., 40 (1993), 17-51.

[46] Serre, D. Solutions faibles globales des quations de Navier-Stokes pour un fluide compressible. C. R. Acad. Sci. Paris Sér. I Math. 303 (1986), no. 13, 639-642.

[47] Serre, D. On the one-dimensional equation of a viscous, compressible, heatconducting fluid. C. R. Acad. Sci. Paris Sér. I Math. 303 (1986), no. 14, 703-706.

[48] Solonnikov, V. A. On solvability of an initial boundary value problem for the equations of motion of viscous compressible fluid. Zap. Nauchn. Sem. LOMI 56 (1976), 128-142.

[49] Vaigant, V. A.; Kazhikhov, A. V. On the existence of global solutions of twodimensional Navier-Stokes equations of a compressible viscous fluid. (Russian) Sibirsk. Mat. Zh. 36 (1995), no. 6, 1283-1316

[50] Valli A.; ZajaczkowskiW. M. Navier-Stokes equations for compressible fluids: global existence and qualitative properties of the solutions in the general case. Comm. Math. Phys. 103 (1986), no. 2, 259-296.

[51] Vong, S.-W.; Yang, T.; Zhu, C. Compressible Navier-Stokes equations with degenerate viscosity coefficient and vacuum. II. J. Differential Equations 192 (2003), no. $2,475-501$.

[52] Xin, Z. Blowup of smooth solutions to the compressible Navier-Stokes equation with compact density. Comm. Pure Appl. Math. 51, 1998, pp. 229-240.

[53] Xin, Z. On the behavior of solutions to the compressible Navier-Stokes equations. 159-170, AMS/IP Stud. Adv. Math. 20, AMS, Providence, RI, 2001.

[54] Xin, Z.; Yuan, H. Vacuum state for spherically symmetric solutions of the compressible Navier-Stokes equations, Journal of Hyperbolic Differential Equaitons $\mathbf{3}$ (2006), 403-442 .

[55] Yang T.; Yao Z.; Zhu, C. Compressible Navier-Stokes equations with densitydependent viscosity and vacuum. Comm. Partial Differential Equations 26 (2001), no. 5-6, 965-981.

[56] Yang, T.; Zhao, H. A vacuum problem for the one-dimensional compressible Navier-Stokes equations with density-dependent viscosity. J. Differential Equations 184, 2002, 163-184.

[57] Yang, T.; Zhu, C. Compressible Navier-Stokes equations with degenerate viscosity coefficient and vacuum. Comm. Math. Phys. 230, 2002, pp. 329-363. 